\title{
Cleaning Up Mechanistic Debris Generated by Twister Ribozymes Using Computational RNA Enzymology
}

\author{
Colin S. Gaines, Timothy J. Giese, and Darrin M. York ${ }^{\star}$ \\ Laboratory for Biomolecular Simulation Research, Institute for Quantitative Biomedicine and \\ Department of Chemistry and Chemical Biology, Rutgers University, Piscataway, NJ 08854, USA
}

\section{Abstract}

The catalytic properties of RNA have been a subject of fascination and intense research since their discovery over 30 years ago. Very recently, several classes of nucleolytic ribozymes have emerged and been characterized structurally. Among these, the twister ribozyme has been center-stage, and a topic of debate about its architecture and mechanism owing to conflicting interpretations of different crystal structures, and in some cases conflicting interpretations of the same functional data. In the present work, we attempt to clean up the mechanistic "debris" generated by twister ribozymes using a comprehensive computational RNA enzymology approach aimed to provide a unified interpretation of existing structural and functional data. Simulations in the crystalline environment and in solution provide insight into the origins of observed differences in crystal structures, and coalesce on a common active site architecture, and dynamical ensemble in solution. We use GPU-accelerated free energy methods with enhanced sampling to ascertain microscopic nucleobase $p K_{a}$ values of the implicated general acid and base, from which predicted activity-pH profiles can be compared directly with experiments. Next, ab initio quantum mechanical/molecular mechanical (QM/MM) simulations with full dynamic solvation under periodic boundary conditions are used to determine mechanistic pathways through multi-dimensional free energy landscapes for the reaction. We then characterize the rate-controlling transition state, and make predictions about kinetic isotope effects and linear free energy relations. Computational mutagenesis is performed to explain the origin of rate effects caused by chemical modifications and make experimentally testable predictions. Finally, we provide evidence that helps to resolve conflicting issues related to the role of metal ions in catalysis. Throughout each stage, we highlight how a conserved L-platform structural motif, to- gether with a key L-anchor residue, forms the characteristic active site scaffold enabling each of the catalytic strategies to come together not only for the twister ribozyme, but the majority of the known small nucleolytic ribozyme classes.

\section{Graphical Abstract}

\footnotetext{
* Darrin.York@ rutgers.edu.

Supporting Information Available

Additional details for computational methods; Descriptions of models used for fitting experimental activity-pH profiles; Optimized parameters and free energies derived from activity-pH models; Radial distribution function and representative snapshots of the active site ion environment as a function of $\mathrm{pH}$; $1 \mathrm{D} \mathrm{QM} / \mathrm{MM}$ free energy profiles for the phosphoryl transfer step; Results from the conformational influencer model and QM/MM free energy calculations for proposed wild-type reaction mechanism; Depiction of theory for kinetic isotope effects. This information is available free of charge on the ACS Publications website.
} 


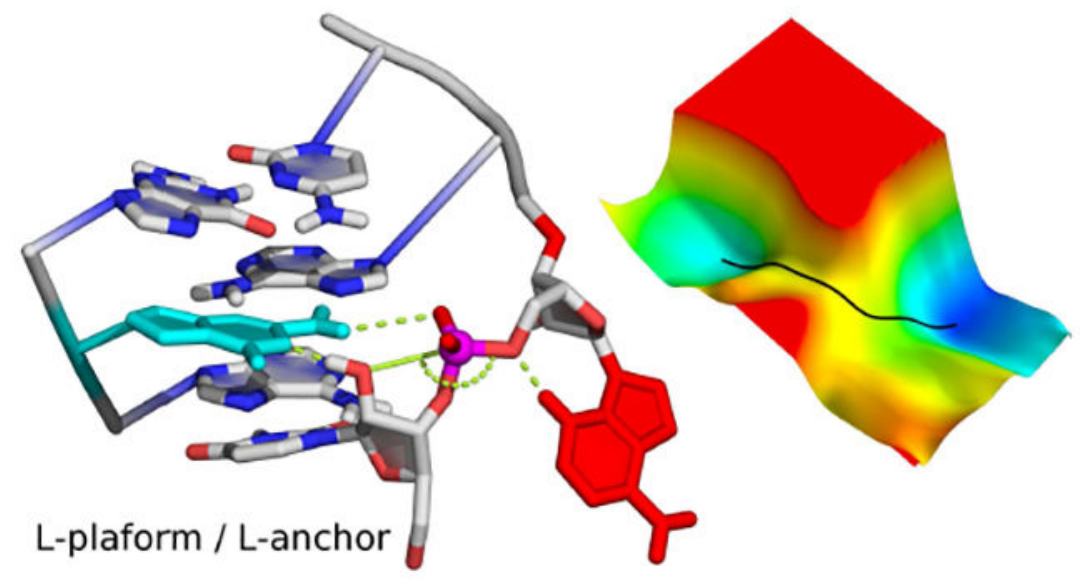

\section{Keywords}

RNA catalysis; twister ribozyme; molecular simulation; free energy; quantum mechanical/ molecular mechanical; kinetic isotope effects; L-platform motif

\section{Introduction}

The mechanisms whereby molecules of RNA can catalyze chemical reactions in biology has been a topic of tremendous interest, and growing impact since its discovery over three decades ago. A predictive understanding of the mechanisms of RNA catalysis in natural biological contexts can ultimately be transferred to synthetic systems such as xeno nucleic acids $^{1}$ or Hachimoji DNA and RNA ${ }^{2}$ that have promise for future therapeutic and synthetic biological applications. Much of what is known about these mechanisms have been gleaned from detailed experimental and computational studies of small nucleolytic ribozymes that catalyze site-specific cleavage (and ligation) of RNA. ${ }^{3-5}$ These ribozymes are widespread in both bacterial and human genomes ${ }^{6-9}$ where they likely play complex roles in RNA processing and regulation of gene expression, and have impact in biotechnology, ${ }_{10,11}$ medicine, ${ }^{12-14}$ and theories into the origins of life itself. ${ }^{15-17}$ In the last 5 years, the number of known naturally occurring nucleolytic ribozyme classes has roughly doubled, sparking a surge of experimental effort aimed toward their structural and functional characterization. 8,9 This wealth of new information promises to reveal new insight into the diverse array of catalytic mechanisms available to RNA, ${ }^{18}$ including common themes and possible evolutionary connections between ribozyme classes.

Among the newly discovered ribozyme classes, the twister ribozyme stands apart as a system that has attracted a great deal of attention, and ignited several debates in the literature. Recently, experts have brought to the forefront a critical barrier to progress in the field: the "mechanistic debris" generated by twister ribozymes, created in the wake of the rush to unveil details of its catalytic mechanism. ${ }^{19}$ This debris arises from diverse biophysical data sets that lead to divergent structural models, conflicting interpretations of essentially the same biochemical data, and inconsistent use of different terms to discuss the same catalytic effects. 
Twister ribozymes catalyze RNA transphosphorylation that leads to site-specific cleavage of the RNA phosphodiester backbone. This is a fundamentally important reaction in biology that is catalyzed by naturally occurring nucleolytic ribozymes (hammerhead, ${ }^{20-22}$ hairpin, ${ }^{23,24} \mathrm{HDV},{ }^{25,26} \mathrm{VS},{ }^{27,28} \mathrm{glmS},{ }^{29,30}$ twister, ${ }^{8,31}$ pistol, $,{ }^{9,32} \mathrm{TS},{ }^{9,33}$ and hatchet ${ }^{9}$ ribozymes), and protein enzymes (e.g., RNase $\mathrm{A}^{34}$ ), as well as artificially engineered DNA enzymes (e.g., 8-17 DNAzyme ${ }^{35}$ ). In this reaction, the $2^{\prime} \mathrm{O}$ makes a nucleophilic attack on the phosphorus atom of the adjacent scissile phosphate to form a pentavalent transition state (or metastable intermediate), followed by departure of the $\mathrm{O}^{\prime}$ leaving group to produce $2^{\prime}, 3^{\prime}$ cyclic phosphate and $5^{\prime} \mathrm{OH}$ cleavage products. In order to achieve catalytic rate enhancements that range from $10^{6}-10^{8}$ for RNA enzymes, ${ }^{36,37}$ and several orders of magnitude greater for RNase $\mathrm{A},{ }^{34}$ enzymes employ up to four mutually enhancing catalytic strategies (designated $a, \beta, \gamma$ and $\delta$ ): 36

- $\quad a$ : Arrangement of the $\mathrm{O}^{\prime}{ }^{\prime}$ nucleophile, $\mathrm{P}$ atom and $\mathrm{O}^{\prime}$ leaving group in an active in-line attack geometry.

- $\quad \beta$ : Electrostatic stabilization (neutralization/protonation) of the negative charge accumulation on the non-bridging phosphoryl oxygens (NPOs) at the pentavalent transition state.

- $\quad \gamma$. Activation (deprotonation) of the $2^{\prime} \mathrm{OH}$ to facilitate nucleophilic attack.

- $\delta$ : Stabilization (neutralization/protonation) of the accumulating charge of the $\mathrm{O}^{\prime}$ to facilitate leaving group departure.

In the case of the twister ribozyme, there is strong evidence from functional studies that $\gamma$ and $\delta$ strategies occur through a general base/acid mechanism involving two highly conserved nucleobase residues: the N1 position of G33 and N3 position of A1. ${ }^{38}$ The latter general acid strategy has never before been implicated, as the most likely acid site on an adenine nucleobase is at the $\mathrm{N} 1$ position rather than the $\mathrm{N} 3,{ }^{39}$ due to the unperturbed microscopic $p K_{a}$ of $\mathrm{N} 1$ being significantly closer to neutrality. The proposition of the N3 position of A1 as the general acid was initially met with some controversy, ${ }^{19}$ primarily due to the interpretations of different sets of crystallographic data, and in some cases different interpretations of functional data sets. Further questions remained unanswered, such as: How does the twister ribozyme adopt a catalytically active conformation in solution? What are the origins of the apparent $\mathrm{pK}_{a}$ shifts of the general acid and base, and how are they related to microscopic $\mathrm{pK}_{a}$ values? What elements serve to stabilize the transition state, and how does this affect bonding? What is the role of solvent components, including divalent metal ions, in the reaction? While new insight into these questions has been provided by recent experiments, ${ }^{38}$ detailed answers must derive from rigorous computational modeling that provides a unified atomic-level interpretation of the current body of experimental data.

Multi-scale modeling/simulation provide powerful tools to aid in the mechanistic interpretation of experimental data on enzymes ${ }^{40,41}$ and ribozymes. ${ }^{42}$ However, it must be remembered that even the most rigorous simulations, in the end, rely on models, and are only meaningful if they are able to explain a broad range of experimental data for the system under study. Here, we apply a comprehensive computational RNA enzymology approach ${ }^{42}$ to clean up the mechanistic debris, as defined previously, generated by twister ribozymes, 
and hopefully open the door to a unified interpretation of the current body of structural and functional data such that a consensus view of mechanism can emerge.

In this approach, we first consider available crystallographic structures to explore the origin of their structural differences, and perform crystal simulations to validate our simulation models and provide a baseline for discussion of predicted rearrangements that occur in solution. We then use molecular dynamics (MD) simulations to derive a structural and dynamical model of the catalytically active conformation and protonation state in solution that is consistent with a wide range of functional data. Using GPU-accelerated free energy methods, we characterize the probability of observing the ribozyme in its active state as a function of $\mathrm{pH}$, and validate the model by comparison with the experimentally measured activity-pH profiles. Departing from the active state, we determine the intrinsic reaction free energy barrier and catalytic pathway for the chemical steps of the reaction using $a b$ initio combined quantum mechanical/molecular mechanical (aiQM/MM) simulations. ${ }^{40-42} \mathrm{We}$ then make experimentally testable predictions of the heavy-atom kinetic isotope effects based on the calculated transition state ensemble. We provide resolution of issues regarding the catalytic role of a divalent metal ion in the active site, and make functional predictions that can be further tested with experimental mutagenesis. Throughout, we discuss the twister ribozyme mechanism using the simplified framework of four fundamental catalytic strategies for RNA transphosphorylation discussed above, and draw important conclusions about how catalytic RNAs exhibit both similarities and fundamental differences to RNAcleaving enzymes in the protein world. Finally, we demonstrate how the combination of all four catalytic strategies are brought together and enabled by the L-platform motif, which forms a characteristic scaffold in the active site of not just the twister ribozyme, but most currently known small nucleolytic ribozyme classes.

\section{Results}

\section{Discrepancies in crystal structures stem from packing that disrupts weak helices}

The recent discovery of the twister ribozyme from comparative genomics ${ }^{8}$ sparked the generation of a wealth of structural data from X-ray crystallography ${ }^{31,43-45}$ that has been discussed in a recent review. ${ }^{46}$ The twister ribozyme secondary structure (Figure 1a) consists of three alternating stems (P1, P2, and P4) and loops (L1, L2, and L4) which are organized into a compact fold (Figure 1b) by the tertiary contacts formed by two pseudoknots T1 and $\mathrm{T} 2$. The scissile phosphate contained within the L1 loop is then positioned in the center of the ribozyme where, in addition to stacking interactions, a series of non-standard (i.e. not canonical WC) base pairs and hydrogen bonds form the active site (Figure 1d). The architecture of the functional active site is supported by an L-platform/L-anchor motif (Figure 1c) that acts as a central scaffold for positioning key nucleobase residues and enabling all four catalytic strategies to come together.

Currently, there are five different crystal structures (PDB IDs 4QJH, ${ }^{43} 4 \mathrm{OJI},{ }^{31} 4 \mathrm{RGE}^{44}$ and $5 \mathrm{DUN},{ }^{45}$ and $4 \mathrm{QJD}{ }^{43}$ ) four of which are significantly distinct (4RGE and 5DUN differ only in the deactivating mutation, $2^{\prime}$-deoxy and $2^{\prime} \mathrm{O}$-methyl, respectively) available of the twister ribozyme (Figure 2), all of which require a conformational rearrangement to arrive at a catalytically active state. The two major areas of difference in the structures involve the 
folding of the P1 stem, and the position of the uracil (U-1) immediately upstream of the scissile phosphate. A comparative analysis of these crystal structures has been presented by Gebetsberger and Micura, ${ }^{46}$ and below we provide additional insight into the origins of structural differences and their mechanistic interpretation.

Focusing first on the P1 stem, a clear trend emerges: the major discrepancies in the structural models stem from crystal packing that disrupts weak helices. The two sequences that have the longest CG-rich P1 stems, 4QJH (Figure 2a) and 4OJI (Figure 2b) with 8 bp and 5 bp, respectively, are fully intact and pack by coaxially stacking in the crystal. Whereas the two sequences 4RGE (Figure 2c) and 4QJH (Figure 2d) with 4 bp stems of exclusively weak base pairs (A-U or G-U) are seen to have P1 stems whose $5^{\prime}$ strand is displaced (partially in the 4RGE and fully in 4QJD) as a result of crystal packing. A further distinction of the 4RGE sequence is that in the crystal U-2 and U-5 form base triples involving trans $\mathrm{WC} / \mathrm{H}$ and cis $\mathrm{WC} / \mathrm{H}$ base pairs with A34 and A35, respectively; the 4QJD sequence with guanines at positions -2 and 35 does not form these additional base pairing interactions with the L1 loop.

Adding to the puzzle, recent FRET studies of both the Oryza sativa ${ }^{47}$ and env22 $2^{48}$ twister ribozymes (corresponding to the 4OJI and 4RGE structures, respectively) provided conflicting evidence for the importance of the phylogenetically conserved P1 stem in the folding the ribozyme. For the 4OJI sequence, folding of the critical T2 pseudoknot was correlated with a fully intact and strongly base paired P1 stem, while for the 4RGE sequence the P1 stem is neither essential for folding nor efficient cleavage activity (as show by studies where the P1 stem was eliminated in its entirety ${ }^{45}$ ). As will be discussed below, this data is reconciled by considering how a misfolded P1 stem may interfere with local conformational rearrangements that result in the formation of the essential L-platform motif within the active site.

Moving now to the active site contained within the L1 loop immediately adjacent to the P1 stem, it is observed that $\mathrm{U}-1$ is extruded from any helical stacking in every structure. Further, none of the crystal structures, except 4RGE, have the U-1 residue positioned such that the $\mathrm{O} 2$ ' nucleophile would be reasonably in-line with the scissile phosphate. However, computational studies ${ }^{49}$ departing from the 4RGE structure suggest that, in solution, in-line fitness is not maintained and an active state is not achieved. Given that in-line attack by $\mathrm{U}-1: \mathrm{O}^{\prime}$ is essential for catalysis, it is clear that some local conformational rearrangement is required for each crystal structure to form a catalytically active state in solution.

Toward this end, we depart from the 4OJI structure, which is not only the highest resolution structure at $(2.3 \AA)$, but also has strong base pairing in the P1 stem that appears less perturbed by crystal packing compared to the other crystal structures. In order to validate our simulation models, we performed simulations in the crystalline environment to compare the structure and fluctuations directly to crystallographic data (Figure 3). The simulated and experimental structures were overall very close (RMSD 0.80 $\mathrm{A}$ ), as were the B-values (RMSE $12.98 \AA^{2}, R=0.92$ ). These results lent credence to our simulation models and bolstered confidence in our prediction of the conformational rearrangements (described in the next section) that occur in solution resulting from removal of crystal packing restraints. 


\section{A local conformational rearrangement is required to form a catalytically active state in solution}

In the 4OJI crystal structure, U-1 is observed to be involved in a crystal packing contact with G14' (symmetry-related monomer). Crystal simulations (Figure 3b) indicate that hydrogen bonding between $\mathrm{U}-1$ and G14' locks U-1 in an extruded state that prevents in-line fitness (Figure 4a). In solution, the extruded state remains populated, and becomes more flexible in the absence of the packing restraints (Figure $4 \mathrm{~b}$ ). Long-time simulations reveal that the twister active site can undergo a local conformational rearrangement whereby U-1 stacks under G33 and forms a tWH/tWW (U-1/A34/A19) base triple that enables good in-line fitness to be achieved. ${ }^{50}$ There exists an intermediate conformational state between the extruded and triple states, whereby U-1 stacks with G33 but is not within hydrogen bonding distance of A34 that also sufficiently enables in-line alignment of the nucleophile with the scissile phosphate. Over these unrestrained long-time simulations ${ }^{50}$ all three states are sampled, demonstrating the conformational flexibility of the U-1 residue in solution relative to in the crystal structures. Connecting back to the discussion of the structural data, the positioning of the U-1 residue at the heel of the L-platform provides a model that can begin to unify the interpretation of these experiments. For the positioning of the inherently flexible U-1 residue in the base triple (U-1/A34/A19 and stacked with G33) to occur, the Hoogsteen edge of A34 must be accessible. The evidence suggests that this can be achieved either with a strongly base paired P1 stem, as seen with the Oryza sativa ribozyme or by elimination of the P1 stem to prevent U-2 from competing for the triple with A34 and A19 observed with the env22 construct.

With U-1 in the base triple (with G33 and A1 nucleobases in their standard/neutral protonation states), significant sampling of conformations where the nucleophile poised for in-line attack (Figure 4c) is observed. When the general base and acid residues are in their active protonation states $\left(\mathrm{G} 33: \mathrm{N1}^{-}\right.$and $\left.\mathrm{A} 1: \mathrm{N} \mathrm{H}^{+}\right)$, there is a considerable enhancement of the in-line fitness (Figure 4d) that is supported by stable hydrogen bonding with the $\mathrm{O}^{\prime}$ nucleophile and $\mathrm{O}^{\prime}$ leaving group. The same trend is seen with U-1 restrained to stack with G33 without forming the base triple. ${ }^{50}$ It should be noted that in solution the U-1 residue has been seen to interconvert between these conformational states, ${ }^{50}$ and therefore this residue was restrained in order to focus on exploring in-line fitness as a function of conformational and protonation state. In either the "stacked" or base "triple" conformation, G33:N1 is poised to act as the general base to activate the nucleophile, and A1:N3 is positioned to act as a general acid catalyst to donate a proton to the leaving group. In previous work, both the "stacked" and "triple" states have been considered and discussed in detail. ${ }^{50}$ Here, we focus on the more structured U-1/A34/A19 base triple, that together with the active G33:N1-/ A1: $\mathrm{N} \mathrm{H}^{+}$protonation state (Figure $4 \mathrm{~d}$ define the "active state" of the ribozyme. This state is used later as and departure point for aiQM/MM simulations of the chemical steps of the reaction. The observed rate of cleavage is then directly proportional to the probability of observing the ribozyme in its active state. Characterization of this probability is challenging since both protonation and conformation state elements are coupled to one another and strongly dependent on $\mathrm{pH}$ and ionic conditions. In the next two sections we consider each of these states and explore the nature of their coupling. 


\section{Interpretation of activity-pH and $\mathrm{pK}_{a}$ data}

Activity-pH datasets have been collected for a 'wild-type' bimolecular type P3 twister ribozyme construct and a variety of mutants over a wide range of $\mathrm{pH}$ values under ion concentrations of $10 \mathrm{mM} \mathrm{MgCl}_{2}, 100 \mathrm{mM} \mathrm{NaCl}$ and $0.05 \mathrm{mM}_{\text {EDTA. }}{ }^{31,38}$ Additionally, NMR measurements for the $\mathrm{pK}_{a}$ of the presumptive general acid (A1) have been collected by measuring the chemical shift of ${ }^{13} \mathrm{C} 2$-labeled-A1 both as part of a bimolecular twister construct as well as the substrate strand alone. ${ }^{45}$ For the twister complex, the measured microscopic $p K_{a}$ is 5.1 , and likely corresponds to the catalytic N3 site (although the experiment is unable to distinguish between protonation at either N1 or N3). Similar attempts were made to measure the microscopic $\mathrm{pK}_{a}$ of the general base (G33), but were hindered by instability of the RNA at $\mathrm{pH}$ values above 9.5; however, the current body of evidence suggests the $\mathrm{pK}_{a}$ of $\mathrm{G} 33$ is unshifted toward neutrality in the ribozyme. ${ }^{45}$

To aid in the interpretation of the experimental activity-pH data, we consider a series of three successively more complex models: ${ }^{51}$ 1) A simple, non-cooperative model, 2) a cooperative model that allows coupling of general acid and base protonation states, and 3) an influencer model that further couples protonation and conformational states. The free energy differences for each leg of the thermodynamic cycle (Figure S1) corresponding to the cooperative model were calculated in an effort to estimate both the microscopic $\mathrm{pK}_{a}$ values for the general acid and base in the ribozyme environment and the coupling between them. The predicted $\mathrm{pK}_{a}$ values for $\mathrm{A} 1: \mathrm{N} 3$ and G33:N1 are $5.75 \pm 0.23$ and $9.24 \pm 0.18$, respectively, with a the coupling between them $\left(\Delta p K_{\text {coop }}\right)$ of 0.21 . While these estimates are reasonably consistent with the spectroscopic values, we opted to use the data from our simulations in the most conservative fashion. Therefore, the experimental $\mathrm{pK}_{a}$ values were used as constraints, in addition to the calculated coupling, such that each of these models has three "free" parameters (Table S1) used to fit the data with the same statistical quality $\left(\mathrm{R}^{2}=\right.$ 0.9894), and are described in detail in the Supporting Information.

The only model that is able to fit the activity-pH data ${ }^{31,38}$ with apparent $\mathrm{pK}_{a}$ values of the general acid and base (6.8 and 9.5, respectively) while accounting for the microscopic $\mathrm{pK}_{a}$ values from NMR measurements (5.1 and 9.5, respectively) and predicting responses to mutational effects, is the conformational influencer model shown in Figure 5a. This model predicts that the rate constant associated with the $\mathrm{pH}$-independent rate of cleavage (a function of both the intrinsic rate and any other $\mathrm{pH}$ independent behavior), $\mathrm{k}_{c l}$, is $\geq 200,000$ $\mathrm{min}^{-1}$, corresponding to a barrier of $\leq 12.6 \mathrm{kcal} / \mathrm{mol}$. It is important to note that the activity$\mathrm{pH}$ data considered in this analysis ${ }^{31,38}$ was collected for a twister construct with guanine at the -1 position, rather than the strongly preferred uracil (90\% conserved identity). ${ }^{8}$ This mutation was necessary for accurate kinetics measurements as the sequences with uracil cleaved too rapidly to be accurately measured (T. J. Wilson and D. M. J. Lilley, personal communication). As such, the estimate for the intrinsic barrier for the wild type twister ribozyme with uracil at the -1 position is expected to be less than $12.6 \mathrm{kcal} / \mathrm{mol}$, the upper bound predicted for the $\mathrm{pH}$-independent rate of the G-1 mutant.

In addition to explaining a broader range of experimental data, our simulations provide support for the conformational influencer model. As demonstrated above, and in previous work, ${ }^{50}$ the positioning of $\mathrm{U}-1$ at the heel of the L-platform is critical to forming a 
catalytically active state in solution. Therefore, we hypothesize that a $\mathrm{pH}$-dependent equilibrium between the extruded and triple states (or at the very least stacking of U-1 with G33) of U-1 may provide the underlying physical basis for the conformational influencer. Given that this proposed model for the active conformation of U-1 is characterized not only by hydrogen bonding with A34, but also by stacking G33, this hypothesis could be tested with 2-aminopurine fluorescence spectroscopy. Twister ribozyme constructs that have a weak base pairing P1 stem or no P1 stem at all have already been shown to cleave with 2aminopurine as the -1 residue with only a mild decrease in the observed rate. ${ }^{45}$ Therefore, it is reasonable to propose extending these fluorescence experiments to explore the $\mathrm{pH}$ dependence with the twister ribozyme construct that contains a strongly base paired P1 stem (and for which the kinetics data was collected and subsequent computational modeling was performed), as they could directly assess whether the local rearrangement that completes the L-platform is in fact the conformational influencer. In any event, this conformational influencer model enables the consistent interpretation of the currently available experimental data and, when combined with our simulations, establishes a model for the active state in solution that serves as a departure point to further probe the catalytic chemical steps of the reaction.

\section{Quantum free energy simulations predict stepwise nucleophile activation followed by a concerted mechanism of nucleophilic substitution with partial proton transfer in the rate- controlling transition state}

In studying the twister ribozyme chemical mechanism, we consider the general reaction scheme:

$$
E+\mathrm{S} \stackrel{\Delta G_{b}}{\rightleftharpoons} \mathrm{ES}_{\mathrm{u}} \stackrel{\Delta G_{f}}{\rightleftharpoons} E S_{f} \stackrel{\Delta G_{\text {active }}}{\rightleftharpoons} \mathrm{ES}_{\mathrm{r}}^{*} \rightarrow[E S]_{1}^{\ddagger} \rightarrow \mathrm{ES}_{\mathrm{AP}} \rightarrow[E S]_{2}^{\dagger} \rightarrow E P \stackrel{\Delta G_{b, p}}{\rightleftharpoons} \mathrm{E}+\mathrm{P}
$$

where "E", "S" and "P" represent the enzyme, substrate, and product, respectively, the subscripts " $u$ " and "f" represent unfolded and folded states, respectively. In the case of the self-cleaving twister ribozyme, we omit discussion of substrate binding and product release (shown in gray), and depart from the folded ground state (ESf). This folded state is in equilibrium with the rarely populated, reactant active state $E S_{r}^{*}$ that is catalytically competent to carry out chemistry. The reaction then proceeds through a first transition state $[E S]_{1}^{\ddagger}$ corresponding to activation of the $2^{\prime} \mathrm{OH}$ nucleophile by the general base to arrive at an "activated precursor" $\left(E S_{A P}\right)$ intermediate. From the $E S_{A P}$ intermediate, the reaction follows a pathway proceeding through a second transition state $[E S]_{2}^{+}$to arrive at the $2^{\prime}, 3^{\prime}$-cyclic phosphate and $5^{\prime} \mathrm{OH}$ product $(E P)$.

In the previous section, we used molecular dynamics and alchemical free energy simulations together with activity-pH and NMR data to establish bounds for the experimentally estimated free energy for forming the active state in solution at optimal $\mathrm{pH}\left(\Delta G_{a c t i v e} \geq 6.18\right.$ $\mathrm{kcal} / \mathrm{mol}$ ) and the $\mathrm{pH}$-independent free energy barrier that includes the rate-controlling chemical step of the reaction $(\leq 12.61 \mathrm{kcal} / \mathrm{mol})$. In the present section, we use multiscale 
quantum mechanical simulations to explore the free energy surfaces corresponding to the chemical steps of the reaction, enabling prediction of pathways and free energy barriers, and providing an atomic-level interpretation of mechanism. ${ }^{40,41}$ Specifically, we use ab initio combined quantum mechanical/molecular mechanical simulations ${ }^{40-42}$ with rigorous longranged electrostatics under full periodic boundary conditions ${ }^{52}$ to determine $2 \mathrm{D}$ free energy profiles $^{53}$ along relevant reaction coordinates (see Methods for details). Similar aiQM/MM methods have been applied very recently to gain insight into mechanisms of phosphoryl transfer in RNA polymerase II. ${ }^{54}$ For the twister ribozyme, we consider three general reaction coordinates (Figure 5b): a phosphoryl transfer, general base and general acid reaction coordinate. The phosphoryl transfer coordinate $\left(R_{3}-R_{4}\right)$ is the difference of the P$\mathrm{O} 5^{\prime}$ leaving group $\left(R_{3}\right)$ and $\mathrm{O}^{\prime}$-P nucleophile attack $\left(R_{4}\right)$ distances. Analogously, the general base $\left(R_{1}-\mathrm{R}_{2}\right)$ and acid $\left(R_{5}-R_{6}\right)$ coordinates are the corresponding difference distances involving proton transfer from the nucleophile to G33:N1, and from A1:N3 to the leaving group, respectively. We consider separately coupling of the phosphoryl reaction coordinate with the general base (Figure 5c) and general acid (Figure 5d and Table S2) coordinates.

As discussed earlier, MD simulations predict both a "stacked" and base "triple" state that exhibit high in-line fitness. ${ }^{50}$ The "stacked" state lacks the tWH base pairing interaction between U-1 and A34 present in the base triple, making the former more conformationally dynamic. As discussed earlier, we have adopted the base triple conformation, along with the active $\mathrm{G} 33: \mathrm{N} 1-/ \mathrm{A} 1: \mathrm{N} \mathrm{H}^{+}$protonation state, as the departure point for aiQM/MM simulations. In order to map out the free energy profiles for the chemical steps of the reaction, many computationally intensive aiQM/MM simulations need to be carried out, and each of these simulations are conducted over much shorter time scale than the long-time classical MD simulations used to study the conformational dynamics of the system. As such, the more structured base triple state is better suited as a departure point for the aiQM/MM simulations. Further, tests of the initial (general base) step of the reaction departing from both the stacked and base triple states indicate that the free energy barrier for nucleophile activation is $\sim 1 \mathrm{kcal} / \mathrm{mol}$ lower for the base triple than the stacked state.

The first free energy profile (Figure 5c) shows the coupling of the general base activation (Yaxis) with progression of phosphoryl transfer (X-axis) along the minimal free energy pathway. It is clear that the general base activation of the nucleophile is essentially fully complete prior to the initial nucleophilic attack of the phosphoryl transfer. This is evident from the vertical line in the 2D map that connects $\mathrm{ES}_{\mathrm{r}}^{*} \rightleftharpoons[\mathrm{ES}]_{1}^{\ddagger} \rightleftharpoons \mathrm{ES}_{\mathrm{AP}}$ and indicates this step occurs with no contribution from the phosphoryl transfer coordinate. From linear free energy relations, ${ }^{55}$ this would correspond to a Brønsted coefficient, $\beta \simeq 1$, resulting from activation of the nucleophile in a pre-equilibrium step. Given the stepwise nature of this part of the reaction, general base activation of the nucleophile can be separated from phosphoryl transfer progression, and this step makes an additive free energy contribution of 2.29 $\mathrm{kcal} / \mathrm{mol}$ to arrive at the activated precursor intermediate $\left(E S_{A P}\right)$.

The second free energy profile (Figure 5d) shows the coupling of proton donate to the leaving group from the general acid (Y-axis) with progression of phosphoryl transfer (X- 
axis) along the minimal free energy pathway. Unlike the general base activation, the general acid and phosphoryl transfer steps are concerted, as indicated by the sigmoidal shape of the minimum free energy path and finite slope at the transition state $[E S]_{2}^{\ddagger}$. The barrier to arrive at the transition state from the $E S_{A P}$ intermediate is $7.34 \mathrm{kcal} / \mathrm{mol}$. The rate-controlling transition state $[E S]_{2}^{\ddagger}$ occurs at a phosphoryl transfer coordinate slightly greater than 0 , indicating $\mathrm{P}-\mathrm{O} 5^{\prime}$ bond cleavage is very slightly more progressed in terms of bond order relative to $\mathrm{O} 2$ ' $-\mathrm{P}$ bond formation, and that these processes are nearly synchronous in the transition state. As will be discussed below, this is in contrast to non-enzymatic RNA cleavage under alkaline conditions and to a lesser extent cleavage catalyzed by RNase A as predicted by theoretical calculations ${ }^{56}$ and supported by kinetic isotope effect measurements. ${ }^{57}$

Combining the free energy estimates for the general base activation and phosphoryl transfer/ general acid steps, the simulations predict an overall intrinsic reaction free energy barrier of $9.63 \mathrm{kcal} / \mathrm{mol}$ to arrive at the rate-controlling transition state departing from the active state $\mathrm{ES}_{\mathrm{r}}^{*} \rightleftharpoons[\mathrm{ES}]_{2}^{\ddagger}$. This is less than the upper bound ( $\leq 12.61 \mathrm{kcal} / \mathrm{mol}$ ) estimated from modeling of the activity-pH data in the previous section. It is not unexpected that the QM/MM simulations of the 4OJI sequence predict that the intrinsic reaction barrier is well below the bound derived from the activity-pH data, because the kinetics were measured with a slower G-1 construct, as discussed previously. The important implication of the calculated free energy barrier falling below the estimated bounds is that the predicted pathway from QM/MM simulations corresponds to a feasible mechanism consistent with experimental constraints.

It should be pointed out that one of the high-resolution crystal structures (4RGE) of Patel and Micura ${ }^{44}$ identifies a $\mathrm{Mg}^{2}+$ ion bound at the active site, whereas the other highresolution structure (4OJI) of Lilley ${ }^{31}$ had an active site devoid of $\mathrm{Mg}^{2}+$ ions. Stereospecific phosphorothioate substitution experiments with thiophilic metal ion rescue have been generated by both of these labs, in addition to the Breaker lab, $, 3,38,45$ and discussed in a recent perspective by Breaker ${ }^{19}$ that concluded the pro- $R$ NPO of the scissile phosphate does not require inner-sphere coordination of a divalent ion for catalysis (but likely is stabilized by hydrogen bonding with the exocyclic amine of G33), consistent with our simulations. The pro-S position also does not appear to require inner-sphere coordination of a metal ion for catalysis as substitution of sulfur at this position has essentially no effect on maximum rate, ${ }^{19}$ although this position does appear to exhibit a modest thiophilic rescue. ${ }^{45}$

Our simulation results provide insight into this important observation. Simulations predict the electrostatic environment created by the active site strongly attracts $\mathrm{Na}+$ ions from solution that interact with both G33:06 and the pro-S NPO of the scissile phosphate, and on average follow the developing charge as it migrates from the nucleophile to the leaving group along the reaction coordinate (Figure S2). In this way, our simulations support the notion that the pro- $S$ NPO may be electrostatically stabilized by metal ions in solution (including $\mathrm{Mg}^{2}+$ as observed in the 4RGE structure ${ }^{44}$ ), but not in such a way that requires direct inner-sphere coordination as confirmed by the phosphorothioate/metal rescue experiments. ${ }^{19}$ It is nevertheless possible that phosphorothioate substitution at the pro-S 
NPO creates a thiophilic divalent ion binding site that modestly enhances the electrostatic stabilization of the transition state ( $\beta$ catalysis), leading to a modest "rescue" enhancement, but is not strictly required for the native substrate. In the next section, we develop experimental tests that can serve to further validate our mechanism and pathway.

\section{Simulation models lead to experimentally testable mutation and kinetic isotope effect predictions}

The molecular simulation models for the active state and catalytic pathway presented here provide a foundation from which to make experimentally testable predictions. Toward that end, we consider a set of mutations to the catalytic nucleobases (G33 and A1) that, to our knowledge have not been measured, and make predictions of how those mutations would affect both the $\mathrm{pH}$-dependent probability of forming the active state and the intrinsic reaction barrier (Figure 6, top). Here we propose chemically precise nucleobase modifications that shift the $p K_{a}$ of G33:N1 while preserving the hydrogen bond interface at the Watson-Crick edge so as not to directly impact hydrogen bonding within the active site. Changes in the $\mathrm{pH}$-dependent active state probability can be directly predicted from the $\mathrm{pK}_{a}$ shift using the influencer model fit to the activity-pH data. Changes in the intrinsic rate can be determined from repeating QM/MM simulations of the relevant steps of the reaction and measuring the free energy differences.

The G33(7cG) mutation (i.e., 7-deazaguanine-33) shifts the $\mathrm{pK}_{a}$ of $\mathrm{N} 1$ up by $0.9 \mathrm{pK}$ units, lowering the probability of the active protonation state and thus raising the $\Delta \mathrm{G}$ for adopting the active state by $1.5 \mathrm{kcal} / \mathrm{mol}$. However, by shifting the $\mathrm{pK}_{a}$ of the general base up, away from neutrality, the difference between its $\mathrm{pK}_{a}$ and that of the nucleophile is reduced. This is reflected in a slightly lower free energy difference between the $E S_{r}^{*}$ and $E S_{A P}$ states and a reduction of the intrinsic barrier by $0.24 \mathrm{kcal} / \mathrm{mol}$. The end result is a predicted $k_{\text {obs }} \leq 93$ $\mathrm{min}^{-1}$ or a 8.5 -fold reduction in the rate constant compared to the wild type twister ribozyme. Similar competing effects are seen with the G33(6sG) mutation (i.e., 6thioguanine-33), with the $\mathrm{pK}_{a}$ being shifted towards neutrality by $1.1 \mathrm{pK}$ units instead of towards the nucleophile. The increased probability of the active protonation state is overshadowed by a significant increase in the free energy associated with the intrinsic rate, leading to a predicted 9-fold decrease in the observed rate. These modest perturbations to the $\mathrm{pK}_{a}$ of the general base, correspond to modest decreases in the predicted rate constant. As an additional validation of our model, a more extreme shift in the $\mathrm{pK}_{a}$ (-5.9 units) of the general base was tested, via mutation to 2 -aminopurine. our prediction of a 63 -fold reduction in $k_{o} b s$ matches closely with the experimentally measured value, at $\mathrm{pH} 7$, corresponding to a 72 -fold decrease in the rate. ${ }^{31}$

The interpretation of the predictions of the intrinsic barrier for the mutations of the general acid are more straightforward compared to the general base. The A1(3cA), general acid knockout mutation results in a predicted intrinsic barrier of roughly $30 \mathrm{kcal} / \mathrm{mol}$ (Figure 6, top). An intrinsic barrier of this magnitude is on par with the background rate of cleavage. ${ }^{58}$ When this mutation measured experimentally ${ }^{38}$ the rate is four orders of magnitude slower at neutral $\mathrm{pH}$ and undetectable at low $\mathrm{pH}$, as predicted from the simulations. Further, $\mathrm{A} 1(7 \mathrm{cA})$ mutation led to enhanced activity and a shift in the activity-pH profile aligned with 
the expected microscopic $\mathrm{pK}_{a}$ shift of the $\mathrm{N} 1$ and $\mathrm{N} 3$ endocyclic nitrogens. We performed $\mathrm{QM} / \mathrm{MM}$ simulations with the $\mathrm{A} 1(3 \mathrm{cA})$ knockout mutation and recapitulated the expected activity loss, and then repeated simulations with a $\mathrm{A} 1(3 \mathrm{cA})$ knockout in addition to a $5^{\prime}$ thio enhanced leaving group chemical modification, which was predicted to rescue activity (Figure 6).

As a final prediction, kinetic isotope effects (KIEs) for the WT mechanism of the twister ribozyme were calculated (Figure 6, bottom). KIEs report on the relative reaction rate constants $\mathrm{k} / \mathrm{k}^{\prime}$ between isotopologues where $k$ and $k^{\prime}$ are the pseudo first-order rate constants for the light and heavy isotopologues, respectively. ${ }^{60}$ Measurement of KIEs offer the most sensitive mechanistic probe of changes in bonding that occurs in proceeding from the reactant state minimum through the rate controlling transition state. KIEs arise from subtle nuclear quantum effects that are responsive to changes in electronic potential energy surfaces and especially bond order, and typically require computational approaches to provide a meaningful atomic-level interpretation. ${ }^{57,61-63}$

Phosphoryl transfer reaction mechanisms have been studied extensively with KIEs, ${ }^{57,60-63}$ particularly with ${ }^{18} \mathrm{O}$ isotope substitution at the nucleophile and leaving group positions ( $1^{\circ}$ isotope effects), and non-bridge phosphoryl oxygen ( $2^{\circ}$ isotope effect positions). The most straight-forward interpretation of these KIEs (Figure S4) is that if the bonding environment of an isotopologue becomes more "loose" in proceeding from reactant to transition state (e.g., if the average bond order associated with an isotopic position decreases), differences in the zero-point energy will cause the reaction to be slower for the heavier isotopologue, leading to a "normal" KIE value $\left(k / k^{\prime}>1\right)$. Conversely, if progression to the transition state leads to a more "tight" bonding environment, this will lead to an "inverse" KIE value $\left(k / k^{\prime}\right.$ $<1)$.

The RNA cleavage ( 2 '-O-transphosphorylation) reaction catalyzed by the twister ribozyme is also catalyzed by the protein enzyme RNase A. ${ }^{34}$ Recently, KIEs have been measured/ calculated for the uncatalyzed reaction ${ }^{61,64}$ as well as catalyzed by RNase $A,{ }^{57,62,65}$ and in the presence of $\mathrm{Zn}^{2}+$ ions. ${ }^{63,66}$ These reactions proceed via a largely associative mechanism, as is typical for transesterification and hydrolysis of phosphate diesters, ${ }^{57,60,67}$ involving nucleophilic attack of the $\mathrm{O} 2^{\prime}$ that proceeds through a dianionic pentavalent transition state. The large inverse $\mathrm{O}^{\prime}$ and large normal $\mathrm{O}^{\prime} 1^{\circ}$ KIEs for the uncatalyzed reaction under alkaline conditions have been interpreted to suggest a considerably late transition state characterized by an almost fully formed $\mathrm{O}^{\prime}$-P bond and an almost fully broken P-O5' bond. Catalysis by RNase A leads to a less pronounced inverse $\mathrm{O}^{\prime}{ }^{\prime}$ and normal $\mathrm{O}^{\prime} \mathrm{KIEs}$, and a transition state characterized by less $\mathrm{P}_{-} \mathrm{O}^{\prime}{ }^{\prime}$ bond cleavage and partial proton transfer from the general acid His119 to the $\mathrm{O}^{\prime}$ leaving group. Catalysis by $\mathrm{Zn}^{2}+$ ions has a similar KIE signature. ${ }^{63}$

In the case of twister ribozyme, we predict even less pronounced inverse $\mathrm{O}^{\prime}{ }^{\prime}$ and normal $\mathrm{O5}^{\prime}$ KIEs than in RNase A, corresponding to a transition state that has a slightly less fully formed $\mathrm{O} 2$ ' $-\mathrm{P}$ bond, and less fully broken $\mathrm{P}-\mathrm{O} 5{ }^{\prime}$ bond with significant degree of proton transfer to the leaving group. The explanation for this prediction is fairly simple when put into the context of general acid catalysis. In RNase A, the general acid is a histidine residue 
with unshifted $p K_{a}$ of around $6 .{ }^{68}$ In twister, the general acid is the N3 position of an adenine residue which is much more acidic (unshifted $\mathrm{pK}_{a}$ less than 2 ). The greater acidity of the general acid in twister ribozyme makes it more reactive, causing the proton transfer to the leaving group to occur more readily and thus earlier along the reaction coordinate. Consequently, the twister ribozyme catalyzed reaction has less $\mathrm{O}^{\prime}-\mathrm{P}$ bond formation and less $\mathrm{P}-\mathrm{O} 5^{\prime}$ bond cleavage, and more advanced proton transfer in the transition state relative to that of the reaction catalyzed by RNase A.

\section{Discussion}

Overall, the computational results presented here provide a detailed dynamical model of twister ribozyme catalysis that unifies the interpretation of the current body of structural and functional data, and makes several experimentally testable predictions. Like other nucleolytic ribozymes, the twister ribozyme catalyzes RNA cleavage with an impressive rate enhancement relative to the uncatalyzed background rate, nearly on par with its protein enzyme counterparts such as RNase A that have evolved to promote multiple turn-over reactions. A striking feature is that this rate enhancement arises from a fast (RNase A-like) intrinsic cleavage rate counterbalanced by slow (low probability) formation of the catalytically active state itself.

The observed rate constant for UpA bound RNase A is $8.4 \times 10^{4} \mathrm{~min}^{-2}$ (approximate activation free energy, $\Delta \mathrm{G}^{\ddagger} \approx 13.2 \mathrm{kcal} / \mathrm{mol}$ ), which is at least 2 orders of magnitude faster than the estimated maximum rate constant for twister. ${ }^{69}$ In order to estimate the probability of the active state, molecular simulations of RNase A at constant $\mathrm{pH}$ have been used to interpret the activity-pH profiles, and have led to the conclusion that there is minimal cooperativity between protonation states of the general base and acid in this system. ${ }^{68}$ Using a simple, non-cooperative model for RNase A with apparent $\mathrm{pK}_{a}$ values of 4.88 and 6.95, adoption of the catalytically active state $E S_{f} \rightleftharpoons E S_{r}^{*}$ represents $2.83 \mathrm{kcal} / \mathrm{mol} .{ }^{70}$ The intrinsic rate according to this model would correspond to a $\Delta \mathrm{G}$ value of $10.3 \mathrm{kcal} / \mathrm{mol}$, which is on par with the estimates for the twister ribozyme. A similar analysis for the VS ribozyme ${ }^{4}$ shows that this same balance is critical for the mechanism of that ribozyme. Even with imperfectly tuned microscopic $p K_{a}$ values for the acid and base (at least two units removed from neutrality in the case of twister), the predicted intrinsic rate of these RNA enzymes is comparable to that of the protein enzyme analog RNase A.

Additionally, as a valuable compliment, the work of Swiderek et al. presents estimates for the "intrinsic rate" of the twister ribozyme for a mechanism departing directly from the crystallographically observed (most probable, but catalytically inactive) state. ${ }^{71}$ Their explored mechanism relies on using one of the NPOs as proton shuttle, since in the most probable conformational and protonation state, neither G33 nor A1 are poised to act in a catalytic role. The calculated intrinsic barrier to reaction for this mechanism is $\sim 30 \mathrm{kcal} / \mathrm{mol}$. This is equivalent to the uncatalyzed/background rate of cleavage,${ }^{58}$ further highlighting the need for the ribozyme to adopt an improbable but catalytically active state in solution.

The realization that the catalytically active state of ribozymes often may be highly improbable demands caution in the interpretation of structural data. X-ray crystal structures 
of nucleolytic ribozymes have been critically important to the field in advancing our understanding of mechanism. However, since X-ray data depict static structures of deactivated ribozymes in crystalline environments, the degree to which they represent a dynamic active state in solution is, at best, speculative. In the case of the twister ribozyme, all of the available crystal structures require at least a local re-arrangement in order to adopt a catalytically active conformation. As discussed previously, molecular simulations ${ }^{49}$ and quantum mechanical calculations ${ }^{71}$ departing from the crystal structure and not realizing a catalytically active state led to negative or conflicting results. Indeed, the recent review by Breaker outlining "mechanistic debris generated by twister ribozymes"19 warns that theoretical investigations departing from disparate structural models yield different predictions about mechanism, and thwart efforts to unify conclusions. The present study thus pays special attention, and indeed sheds light on, the origin of the differences in the currently available X-ray crystal structures (e.g., weak base pairing that can lead to disruption of the P1 stem in the crystal), and goes on to identify a local conformational rearrangement that leads to a catalytically active state that is consistent with known general catalytic strategies. ${ }^{36}$

\section{Methods}

The present work takes a comprehensive computational RNA enzymology approach ${ }^{42}$ to study the catalytic mechanism of the twister ribozyme that combines 1) long-time molecular dynamics simulations in both a crystalline environment ${ }^{72}$ and in solution at several stages along the catalytic reaction pathway, 2) GPU-accelerated alchemical free energy simulations, 3) multi-dimensional ab initio combined quantum mechanical/molecular mechanical (QM/MM) simulations, and 4) computational mutagenesis and kinetic isotope effect calculations. All molecular dynamics simulations were performed using the AMBER 18 package, ${ }^{73}$ in particular the GPU-accelerated simulation engine (PMEMD),${ }^{74}$ using the AMBER ff99OL3 RNA force field which includes $a / \gamma^{75}$ and $\chi^{76}$ dihedral modifications to the standard AMBER ff99 force field. ${ }^{77,78}$ The solvent environment was modeled using TIP4P-Ew waters ${ }^{79}$ with ion parameters for both monovalent ${ }^{80}$ and divalent ions ${ }^{81}$ designed for use with this water model. Alchemical free energy simulations were performed using the GPU-accelerated thermodynamic integration(TI $)^{82,83}$ method recently implemented into AMBER 18 by our group. ${ }^{74}$ The ab initio QM/MM simulations were performed using code implemented in-house within a development version SANDER MD program, ${ }^{73}$ and were conducted in explicit solvent under full periodic boundary conditions using the recently introduced ambient potential composite Ewald method ${ }^{52}$ for rigorous long-ranged electrostatics. 2D profiles were analyzed using the 2D variational free energy profile (vFEP) method. ${ }^{53} \mathrm{QM} / \mathrm{MM}$ simulations and kinetic isotope effect calculations were performed using ab initio PBE0/6-31G* density functional quantum model. ${ }^{84,85}$ Simulations were performed using a wide array of national production cyberinfrastructure provided by the NSF, NIH and Rutgers University. Full details for all computations in this work are provided in the Supporting Information.

\section{Supplementary Material}

Refer to Web version on PubMed Central for supplementary material. 


\section{Acknowledgement}

We thank Dr. David M. J. Lilley and Dr. Timothy J. Wilson for enlightening discussion and data sharing throughout the evolution of this project. The authors are grateful for financial support provided by the National Institutes of Health (No. GM107485 and GM62248 to DMY). Computational resources were provided by the Office of Advanced Research Computing (OARC) at Rutgers, The State University of New Jersey, the National Institutes of Health under Grant No. S100D012346 and by the Extreme Science and Engineering Discovery Environment (XSEDE), which is supported by National Science Foundation (No. ACI-1548562 and No. OCI-1053575). Additionally, this research is part of the Blue Waters sustained-petascale computing project, which is supported by the National Science Foundation (awards 0CI-0725070 and ACI-1238993) and the state of Illinois. Blue Waters is a joint effort of the University of Illinois at Urbana-Champaign and its National Center for Supercomputing Applications.

\section{References}

(1). Anosova I; Kowal EA; Dunn MR; Chaput JC; Van Horn WD; Egli M The Structural Diversity of Artificial Genetic Polymers. Nucleic Acids Res. 2016, 44, 1007-1021. [PubMed: 26673703]

(2). Hoshika S; Leal NA; Kim M-J; Kim M-S; Karalkar NB; Kim H-J; Bates AM; Watkins NE; SantaLucia HA; Meyer AJ; DasGupta S; Piccirilli JA; Ellington AD; SantaLucia J; Georgiadis MM; Benner SA Hachimoji DNA and RNA: A Genetic System with Eight Building Blocks. Science 2019, 363, 884-887. [PubMed: 30792304]

(3). Ward WL; Plakos K; DeRose VJ Nucleic Acid Catalysis: Metals, Nucleobases, and Other Cofactors. Chew,. Rev 2014, 114, 4318-4342.

(4). Wilson TJ; Liu Y; Lilley DM J. Ribozymes and the Mechanisms that Underlie RNA Catalysis. Front. Chem. Sci. Eng 2016, 10, 178-185.

(5). Lilley DMJ How RNA Acts as a Nuclease: Some Mechanistic Comparisons in the Nucleolytic Ribozymes. Biochem. Soc. Trans 2017, 45, 683-691. [PubMed: 28620029]

(6). Hammann C; Luptak A; Perreault J; De La Peña M The Ubiquitous Hammerhead Ribozyme. RNA 2012, 18, 871-885. [PubMed: 22454536]

(7). Webb C-HT; Riccitelli NJ; Ruminski DJ; Lupták A Widespread Occurrence of Self-cleaving Ribozymes. Science 2009, 326, 953-953. [PubMed: 19965505]

(8). Roth A; Weinberg Z; Chen AG; Kim PB; Ames TD; Breaker RR A Widespread Self-cleaving Ribozyme Class Is Revealed by Bioinformatics. Nat. Chem. Biol 2014, 10, 56-62. [PubMed: 24240507]

(9). Weinberg Z; Kim PB; Chen TH; Li S; Harris KA; Lünse CE; Breaker RR New Classes of Selfcleaving Ribozymes Revealed by Comparative Genomics Analysis. Nat. Chem. Biol 2015, 11, 606-610. [PubMed: 26167874]

(10). Liu J; Cao Z; Lu Y Functional Nucleic Acid Sensors. Chem. Rev 2009, 109, 1948-1998. [PubMed: 19301873]

(11). Felletti M; Stifel J; Wurmthaler LA; Geiger S; Hartig JS Twister Ribozymes as Highly Versatile Expression Platforms for Artificial Riboswitches. Nature Commun 2016, 7, 1-8.

(12). Zhang Y; Wang J; Cheng H; Sun Y; Liu M; Wu Z; Pei R Conditional Control of Suicide Gene Expression in Tumor Cells with Theophylline-responsive Ribozyme. Gene therapy 2017, 24, 84. [PubMed: 27874855]

(13). Sullenger BA; Nair S From the RNA World to the Clinic. Science 2016, 352, 1417-1420. [PubMed: 27313039]

(14). Kim CM; Smolke CD Biomedical Applications of RNA-based Devices. Curr. Opin. Biomed. Eng 2017, 4, 106-115.

(15). Gilbert W The RNA World. Nature 1986, 319, 618.

(16). Wilson TJ; Lilley DMJ The Evolution of Ribozyme Chemistry. Science 2009, 323, 1436-1438. [PubMed: 19286542]

(17). Kun Ádám,; Szilágyi A; Könnyu B; G. Boza; Zachar I; Szathmáry E The Dynamics of the RNA World: Insights and Challenges. Annals of the New York Academy of Sciences 2015, 1341, 7595. [PubMed: 25735569] 
(18). Seith DD; Bingaman JL; Veenis AJ; Button AC; Bevilacqua PC Elucidation of Catalytic Strategies of Small Nucleolytic Ribozymes from Comparative Analysis of Active Sites. ACS Catal 2018, 8, 314-327.

(19). Breaker RR Mechanistic Debris Generated by Twister Ribozymes. ACS Chem. Biol 2017, 12, 886-891. [PubMed: 28191925]

(20). Prody GA; Bakos JT; Buzayan JM; Schneider IR; Bruening G Autolytic Processing of Dimeric Plant Virus Satellite RNA. Science 1986, 231, 1577-1580. [PubMed: 17833317]

(21). Pley HW; Flaherty KM; McKay DB Three-dimensional Structure of a Hammerhead Ribozyme. Nature 1994, 372, 68-74. [PubMed: 7969422]

(22). Scott WG; Murray JB; Arnold JRP; Stoddard BL; Klug A Capturing the Structure of a Catalytic RNA Intermediate: The Hammerhead Ribozyme. Science 1996, 274, 2065-2069. [PubMed: 8953035]

(23). Buzayan JM; Gerlach WL; Bruening G Nonenzymatic Cleavage and Ligation of RNAs Complementary to a Plant Virus Sattelite RNA. Nature 1986, 323, 349-353.

(24). Rupert PB; Massey AP; Sigurdsson ST; Ferré-D'Amaré AR Transition State Stabilization by a Catalytic RNA. Science 2002, 298, 1421-1424. [PubMed: 12376595]

(25). Sharmeen L; Kuo MY; Dinter-Gottlieb G; Taylor J Antigenomic RNA of Human Hepatitis Delta Virus Can Undergo Self-cleavage. J. Virol 1988, 62, 2674-2679. [PubMed: 2455816]

(26). Ferré-D'Amaré ARZhou K; Doudna JA Crystal Structure of a Hepatitis Delta Virus Ribozyme. Nature 1998, 395, 567-574. [PubMed: 9783582]

(27). Saville BJ; Collins RA A Site-specific Self-cleavage Reaction Performed by a Novel RNA in Neurospora Mitochondria. Cell 1990, 61, 685-696. [PubMed: 2160856]

(28). Suslov NB; DasGupta S; Huang H; Fuller JR; Lilley DMJ; Rice PA; Piccirilli JA Crystal Structure of the Varkud Satellite Ribozyme. Nat. Chew,. Biol 2015, 11, 840-846.

(29). Winkler WC; Nahvi A; Roth A; Collins JA; Breaker RR Control of Gene Expression by a Natural Metabolite-responsive Ribozyme. Nature 2004, 428, 281-286. [PubMed: 15029187]

(30). Klein DJ; Ferré-D'Amaré AR Structural Basis of glmS Ribozyme Activation by glucosamine-6phosphate. Science 2006, 313, 1752-1756. [PubMed: 16990543]

(31). Liu Y; Wilson TJ; McPhee SA; Lilley DMJ Crystal Structure and Mechanistic Investigation of the Twister Ribozyme. Nat. Chew. Biol 2014, 10, 739-744.

(32). Ren A; Vusurovic N; Gebetsberger J; Gao P; Juen M; Kreutz C; Micura R; Patel D Pistol Ribozyme Adopts a Pseudoknot Fold Facilitating Site-specific in-line Cleavage. Nat. Chem. Biol 2016, 12, 702-708. [PubMed: 27398999]

(33). Liu Y; Wilson TJ; Lilley DMJ The Structure of a Nucleolytic Ribozyme that Employs a Catalytic Metal Ion. Nat. Chem. Biol 2017, 13, 508-513. [PubMed: 28263963]

(34). Raines RT Ribonuclease A. Chem. Rev 1998, 98, 1045-1066. [PubMed: 11848924]

(35). Santoro SW; Joyce GF A General Purpose RNA-cleaving DNA Enzyme. Proc. Natl. Acad. Sci. USA 1997, 94, 4262-4266. [PubMed: 9113977]

(36). Emilsson GM; Nakamura S; Roth A; Breaker RR Ribozyme Speed Limits. RNA 2003, 9, 907918. [PubMed: 12869701]

(37). Breaker RR; Emilsson GM; Lazarev D; Nakamura S; Puskarz IJ; Roth A; Sudarsan N A Common Speed Limit for RNA-cleaving Ribozymes and Deoxyri-bozymes. RNA 2003, 9, 949 957. [PubMed: 12869706]

(38). Wilson TJ; Liu Y; Domnick C; Kath-Schorr S; Lilley DMJ The Novel Chemical Mechanism of the Twister Ribozyme. J. Am. Chem. Soc 2016, 138, 6151-6162. [PubMed: 27153229]

(39). Kapinos LE; Operschall BP; Larsen E; Sigel H Understanding the Acid-base Properties of Adenosine: The Intrinsic Basicities of N1, N3 and N7. Chem. Eur. J 2011, 17, 8156-8164. [PubMed: 21626581]

(40). Garcia-Viloca M; Gao J; Karplus M; Truhlar DG How Enzymes Work: Analysis by Modern Rate Theory and Computer Simulations. Science 2004, 303, 186-195. [PubMed: 14716003]

(41). Gao J; Ma S; Major DT; Nam K; Pu J; Truhlar DG Mechanisms and Free Energies of Enzymatic Reactions. Chem. Rev 2006, 106, 3188-3209. [PubMed: 16895324] 
(42). Panteva MT; Dissanayake T; Chen H; Radak BK; Kuechler ER; Giambaşu GM; Lee T-S; York DM Multiscale Methods for Computational RNA Enzymology In Methods in Enzymology; Chen S-J, Burke-Aguero DH, Eds.; Academic Press: Cambridge, MA, 2015; Vol. 553; pp 335-374.

(43). Eiler D; Wang J; Steitz TA Structural Basis for the Fast Self-cleavage Reaction Catalyzed by the Twister Ribozyme. Proc. Natl. Acad. Sci. USA 2014, 111, 13028-13033. [PubMed: 25157168]

(44). Ren A; Kosutic M; Rajashankar KR; Frener M; Santner T; Westhof E; Micura R; Patel DJ In-line Alignment and $\mathrm{Mg} 2+$ Coordination at the Cleavage Site of the env22 Twister Ribozyme. Nat. Commun 2014, 5, 5534-5544. [PubMed: 25410397]

(45). Košutić M; Neuner S; Ren A; Flür S; Wunderlich C; Mairhofer E; Vusurović N; Seikowski J; Breuker K; Höbartner C; Patel DJ; Kreutz C; Micura R A Minitwister Variant and Impact of Residues/Cations on the Phosphodiester Cleavage of This Ribozyme Class. Angew. Chem. Int. Ed 2015, 54, 15128-15133.

(46). Gebetsberger J; Micura R Unwinding the Twister Ribozyme: From Structure to Mechanism. WIREs RNA 2016, 8, 1402.

(47). Panja S; Hua B; Zegarra D; Ha T; Woodson SA Metals Induce Transient Folding and Activation of the Twister Ribozyme. Nat. Chem. Biol 2017, 13, 1109-1114. [PubMed: 28825710]

(48). Vusurovic N; Altman RB; Terry DS; Micura R; Blanchard SC Pseudoknot Formation Seeds the Twister Ribozyme Cleavage Reaction Coordinate. J. Am. Chem. Soc 2017, 139, 8186-8193. [PubMed: 28598157]

(49). Ucisik MN; Bevilacqua PC; Hammes-Schiffer S Molecular Dynamics Study of Twister Ribozyme: Role of $\mathrm{Mg}^{2}$ + Ions and the Hydrogen-bonding Network in the Active Site. Biochemistry 2016, 55, 3834-3846. [PubMed: 27295275]

(50). Gaines CS; York DM Ribozyme Catalysis with a Twist: Active State of the Twister Ribozyme in Solution Predicted from Molecular Simulation. J. Am. Chem. Soc 2016, 138, 3058-3065. [PubMed: 26859432]

(51). Frankel EA; Bevilacqua PC Complexity in $\mathrm{pH}-$ Dependent Ribozyme Kinetics: Dark $\mathrm{pK}_{\mathrm{a}}$ Shifts and Wavy Rate-pH Profiles. Biochemistry 2018, 57, 483-488. [PubMed: 29271644]

(52). Giese TJ; York DM Ambient-Potential Composite Ewald Method for ab Initio Quantum Mechanical/Molecular Mechanical Molecular Dynamics Simulation. J. Chem. Theory Comput 2016, 12, 2611-2632. [PubMed: 27171914]

(53). Lee T-S; Radak BK; Pabis A; York DM A New Maximum Likelihood Approach for Free Energy Profile Construction from Molecular Simulations. J. Chem. Theory Comput 2013, 9, 153-164. [PubMed: 23457427]

(54). Tse CKM; Xu J; Xu L; Sheong FK; Wang S; Chow HY; Gao X; Li X; Cheung PP-H; Wang D; Zhang Y; Huang X Intrinsic Cleavage of RNA Polymerase II Adopts a Nucleobase-independent Mechanism Assisted by Transcript Phosphate. Nat. Catal 2019, 2, 228-235.

(55). Jencks WP A Primer for the Bema Hapothle: an Empirical Approach to the Characterization of Changing Transition-state Structures. Chem. Rev 1985, 85, 511-527.

(56). Huang M; Dissanayake T; Kuechler E; Radak BK; Lee T-S; Giese TJ; York DM A Multidimensional B-Spline Correction for Accurate Modeling Sugar Puckering in QM/MM Simulations. J. Chem. Theory Comput 2017, 13, 3975-3984. [PubMed: 28768099]

(57). Harris ME; Piccirilli JA; York DM Enzyme Transition States from Theory and Experiment. Biochim Biophys Acta 2015, 1854, 1727-1728, PMID: 26302659. [PubMed: 26302659]

(58). Li Y; Breaker RR Kinetics of RNA Degradation by Specific Base Catalysis of Transesterification Involving the $2^{\prime}$-Hydroxyl Group. J. Am. Chem. Soc 1999, 121, 5364-5372.

(59). Harris ME; Piccirilli JA; York DM Integration of Kinetic Isotope Effect Analyses to Elucidate Ribonuclease Mechanism. Biochim. Biophys. Acta 2015, 1854, 1801-1808. [PubMed: 25936517]

(60). Hengge AC Isotope Effects in the Study of Phosphoryl and Sulfuryl Transfer Reactions. Acc. Chem. Res 2002, 35, 105-112. [PubMed: 11851388]

(61). Wong K-Y; Gu H; Zhang S; Piccirilli JA; Harris ME; York DM Characterization of the Reaction Path and Transition States for RNA Transphosphorylation Models from Theory and Experiment. Angew. Chem. Int. Ed 2012, 51, 647-651. 
(62). Gu H; Zhang S; Wong K-Y; Radak BK; Dissanayake T; Kellerman DL; Dai Q; Miyagi M; Anderson VE; York DM; Piccirilli JA; Harris ME Experimental and Computational Analysis of the Transition State for Ribonuclease A-catalyzed RNA 2' $-O$-transphosphorylation. Proc. Natl. Acad. Sci. USA 2013, 110, 13002-13007. [PubMed: 23878223]

(63). Chen H; Piccirilli JA; Harris ME; York DM Effect of $\mathrm{Zn}^{2}+$ Binding and Enzyme Active Site on the Transition State for RNA 2' -O-transphosphorylation Interpreted Through Kinetic Isotope Effects. Biochim. Biophys. Acta, Proteins Proteomics 2015, 1854, 1795-1800.

(64). Weissman BP; Li N-S; York DM; Harris M; Piccirilli JA Heavy Atom Labeled Nucleotides for Measurement of Kinetic Isotope Effects. Biochim. Biophys. Acta 2015, 1854, 1737-1745. [PubMed: 25828952]

(65). Kellerman DL; York DM; Piccirilli JA; Harris ME Altered (transition) States: Mechanisms of Solution and Enzyme Catalyzed RNA 2'-O-transphosphorylation. Curr. Opin. Chem. Biol 2014, 21, 96-102. [PubMed: 25023967]

(66). Zhang S; Gu H; Chen H; Strong E; Ollie EW; Kellerman D; Liang D; Miyagi M; Anderson VE; Piccirilli JA; York DM; Harris ME Isotope Effect Analyses Provide Evidence for an Altered Transition State for RNA $2^{\prime}$-O-transphosphorylation Catalyzed by $\mathrm{Zn}^{2}+$. Chem. Commun 2016, 52, 4462-4465.

(67). Perreault DM; Anslyn EV Unifying the Current Data on the Mechanism of Cleavagetransesterification of RNA. Angew. Chem. Int. Ed 1997, 36, 432-450.

(68). Dissanayake T; Swails JM; Harris ME; Roitberg AE; York DM Interpretation of pH-Activity Profiles for Acid-Base Catalysis from Molecular Simulations. Biochemistry 2015, 54, $1307-$ 1313. [PubMed: 25615525]

(69). delCardayré SB; Raines RT Structural Determinants of Enzymic Processivity. Biochemistry 1994, 33, 6031-6037. [PubMed: 8193116]

(70). Park C; Raines RT Catalysis by Ribonuclease A Is Limited by the Rate of Substrate Association. Biochemistry 2003, 42, 3509-3518. [PubMed: 12653555]

(71). Swiderek K; Marti S; Tunon I Molecular Mechanism of the Site-specific Self-cleavage of the RNA Phosphodiester Backbone by a Twister Ribozyme. Theor. Chem. Acc 2017, 136, 0-0.

(72). Ekesan Ş; York DM Framework for Conducting and Analyzing Crystal Simulations of Nucleic Acids to Aid in Modern Force Field Evaluation. J. Phys. Chem. B 2019, in press., Preprint at 10.1021/acs.jpcb.8b11923.

(73). Case DA; Ben-Shalom IY; Brozell SR; Cerutti DS; Cheatham III TE; Cruzeiro VWD; Darden TA; Duke RE; Ghoreishi D; Gilson MK; Gohlke H; Goetz AW; Greene D; Harris R; Homeyer N; Izadi S; Kovalenko A; Kurtzman T; Lee T; Le-Grand S; Li P; Lin C; Liu J; Luchko T; Luo R; Mermelstein DJ; Merz KM; Miao Y; Monard G; Nguyen C; Nguyen H; Omelyan I; Onufriev A; Pan F; Qi R; Roe DR; Roitberg A; Sagui C; Schott-Verdugo S; Shen J; Simmerling CL; Smith J; Salomon-Ferrer R; Swails J; Walker RC; Wang J; Wei H; Wolf RM; Wu X; Xiao L; York DM; Kollman PA AMBER 18 University of California, San Francisco: San Francisco, CA, 2018.

(74). Lee T-S; Cerutti DS; Mermelstein D; Lin C; LeGrand S; Giese TJ; Roitberg A; Case DA; Walker RC; York DM GPU-Accelerated Molecular Dynamics and Free Energy Methods in Amber18: Performance Enhancements and New Features. J. Chew. Inf. Model 2018, 58, 2043-2050, PMID: 30199633.

(75). Pérez A; Marchán I; Svozil D; Sponer J; Cheatham III TE; Laughton CA; Orozco M Refinement of the AMBER Force Field for Nucleic Acids: Improving the Description of $a / \gamma$ Conformers. Biophys. J 2007, 92, 3817-3829. [PubMed: 17351000]

(76). Zgarbová M; Otyepka M; Sponer J; Mládek A; Banás P; Cheatham III TE; Jurečka P Refinement of the Cornell et al. Nucleic Acids Force Field Based on Reference Quantum Chemical Calculations of Glycosidic Torsion Profiles. J. Chew. Theory Comput 2011, 7, 2886-2902.

(77). Cheatham TE III; Cieplak P; Kollman PA A Modified Version of the Cornell et al. Force Field with Improved Sugar Pucker Phases and Helical Repeat. J. Biomol. Struct. Dyn 1999, 16, 845862. [PubMed: 10217454]

(78). Wang J; Cieplak P; Kollman PA How Well Does a Restrained Electrostatic Potential (RESP) Model Perform in Calculating Conformational Energies of Organic Biological Molecules. J. Comput. Chew 2000, 21, 1049-1074. 
(79). Horn HW; Swope WC; Pitera JW; Madura JD; Dick TJ; Hura GL; Head-Gordon T Development of an Improved Four-site Water Model for Biomolecular Simulations: TIP4P-Ew. J. Chem. Phys 2004, 120, 9665-9678. [PubMed: 15267980]

(80). Joung IS; Cheatham III TE Determination of Alkali and Halide Monovalent Ion Parameters for Use in Explicitly Solvated Biomolecular Simulations. J. Phys. Chem. B 2008, 112, 9020-9041. [PubMed: 18593145]

(81). Li P; Roberts BP; Chakravorty DK; Merz KM Jr. Rational Design of Particle Mesh Ewald Compatible Lennard-Jones Parameters for +2 Metal Cations in Explicit Solvent. J. Chem. Theory Comput 2013, 9, 2733-2748. [PubMed: 23914143]

(82). Lee T-S; Hu Y; Sherborne B; Guo Z; York DM Toward Fast and Accurate Binding Affinity Prediction with pmemdGTI: An Efficient Implementation of GPU-Accelerated Thermodynamic Integration. J. Chew,. Theory Comput 2017, 13, 3077-3084.

(83). Giese TJ; York DM A GPU-Accelerated Parameter Interpolation Thermodynamic Integration Free Energy Method. J. Chem. Theory Comput 2018, 14, 1564-1582. [PubMed: 29357243]

(84). Perdew JP; Ernzerhof M; Burke K Rationale for Mixing Exact Exchange with Density Functional Approximations. J. Chem. Phys 1996, 105, 9982-9985.

(85). Adamo C; Scuseria GE Accurate Excitation Energies from Time-dependent Density Functional Theory: Assessing the PBE0 Model. J. Chem. Phys 1999, 111, 2889-2899. 
A

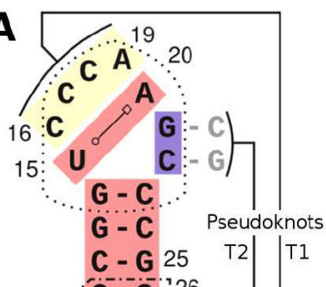

$\mathrm{C}$

A 1

\begin{tabular}{l:c:c}
$A$ & $\mathbf{C} 9$ & $\mathbf{A}$ \\
\hline & $\mathbf{C}$ & $\mathbf{A}^{27}$
\end{tabular}

$\because, G-A$

5 C - G 30

A - U

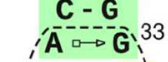

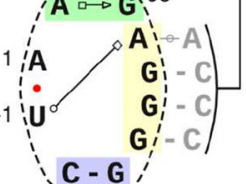

C-G

G - C 40

C- $\mathbf{G}$

C - G

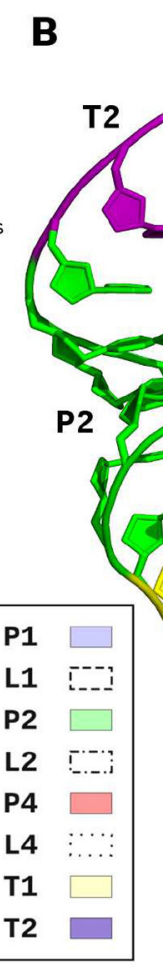

B

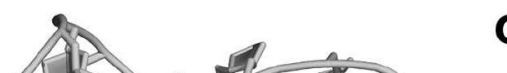

An 1
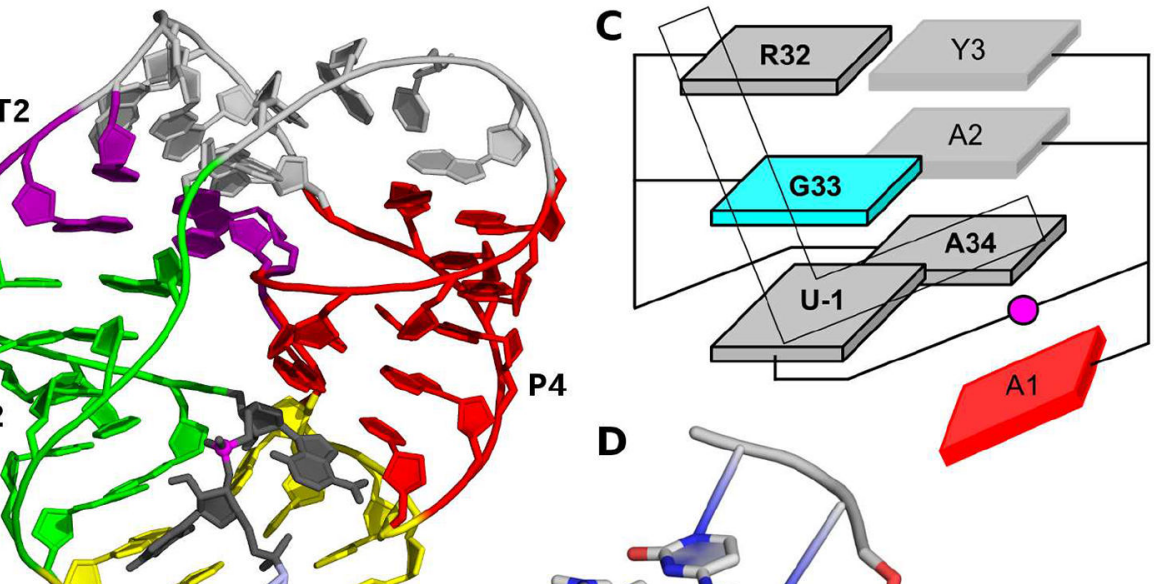

cine

4in

$\rightarrow$

13

2

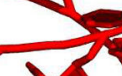

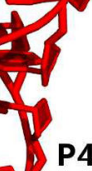

15
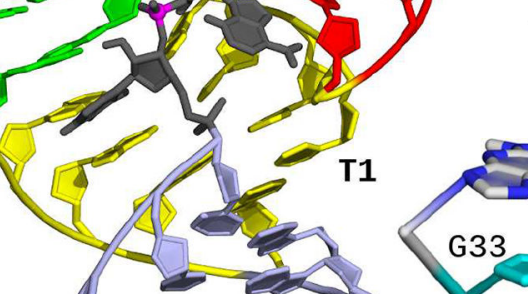

G33

D

$<$

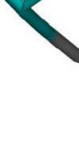

P1

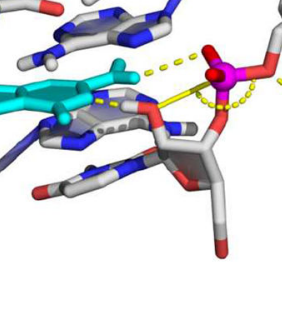

A1

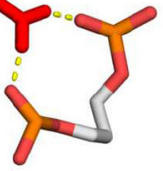

Figure 1: The structure of the twister ribozyme and active site L-platform mo-tif.

(a) The sequence and secondary structure of the twister ribozyme (PDB ID: 4OJI ${ }^{31}$ ),

highlighting secondary structure elements (stems, loops, and pseudoknots) that are directly

comparable across crystallographic structures. (b) Simulation snapshot showing the global fold of the twister ribozyme in a catalytically active state in solution, with color scheme matching that in a. (c) Cartoon-block schematic showing active site base pairing that forms the L-platform motif for the twister ribozyme. The general acid and base are highlighted in red and blue respectively, with the scissile phosphate in magenta. The bolded residues (U-1 and residues 32-34) form the ' $L$ ' of this motif, while $\mathbf{A 2}$ and $\mathbf{Y 3}$ (gray) constitute the 'Lanchor" that serves to anchor the general base. (d) Zoom in of snapshot from $\mathbf{b}$ high-lighting the base pairing and hydrogen bonding around the scissile phosphate characteristic of the Lplatform motif in twister active site. Residues depicted are the same as in $\mathbf{c}$, with the addition of the phosphates of N16/17 shown anchoring A1 in the syn conformation. 


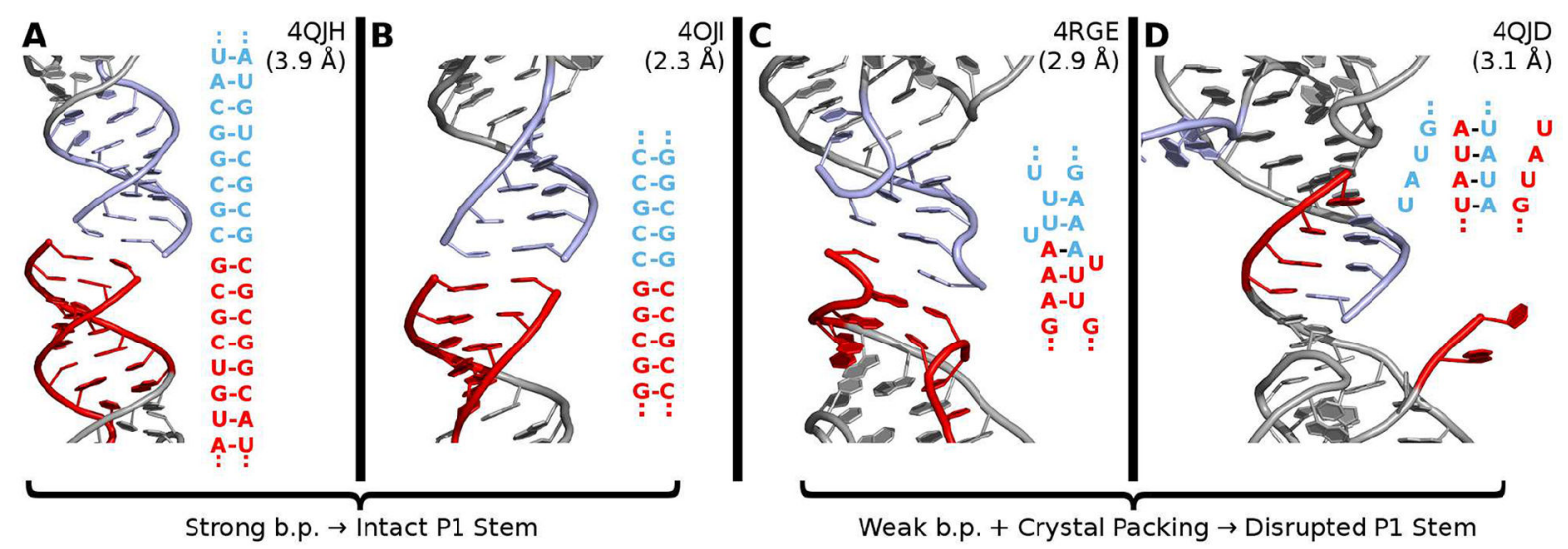

Figure 2: Comparisons of twister ribozyme P1 stem crystal packing.

Cartoon representations of $\mathrm{P} 1$ stems of two symmetry related monomers colored in light blue and red for each of four crystallized sequences. (a) PDB ID: 4QJH ${ }^{43}$ at $3.9 \AA$ A. Eight base pair P1 stem with majority $\mathrm{C}-\mathrm{G}$ pairs remains intact and coaxially stacks in the crystal. (b) PDB ID: $4 \mathrm{OJI}^{31}$ at $2.3 \AA$. P1 stem is comprised of all C-G base pairs, is fully intact and coaxially stacks in the crystal, similar to the 4QJH structure shown in a. (c) PDB ID: $4 \mathrm{RGE}^{44}$ at $2.9 \AA$. Middle two U-A base pairs remain intact, while U-2 and U-4 form base triples in the L1 internal loop. In addition to coaxial stacking, the position of A41 and A41' appear to be an average of two possible orientations for a $\mathrm{WC} / \mathrm{H}$ base pair between those residues. (d) PDB ID: $4 Q \mathrm{QD}^{43}$ at $3.1 \AA$. The $3^{\prime}$ strand of the P1 stem $\left(5^{\prime}-\mathrm{UAUA}^{\prime} 3^{\prime}\right)$ is complementary with the equivalent strand in a symmetry related monomer $\left(3^{\prime}\right.$-AUAU- $\left.5^{\prime}\right)$, leading to base pairing across the monomers. 
A

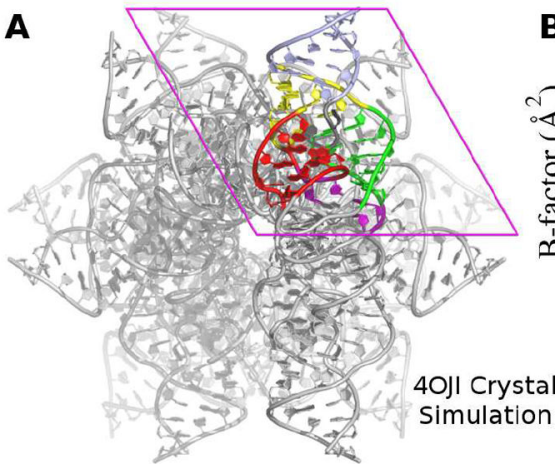

C

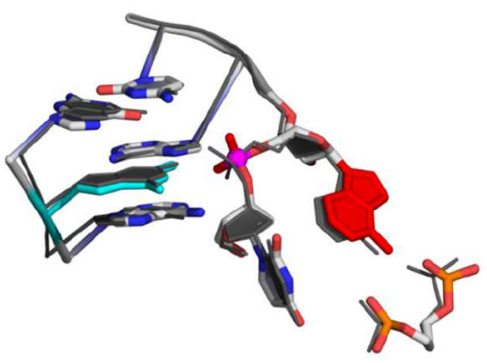

Full Structure RMSD: $0.80 \AA$ L-platform RMSD: $\quad 0.52 \AA$
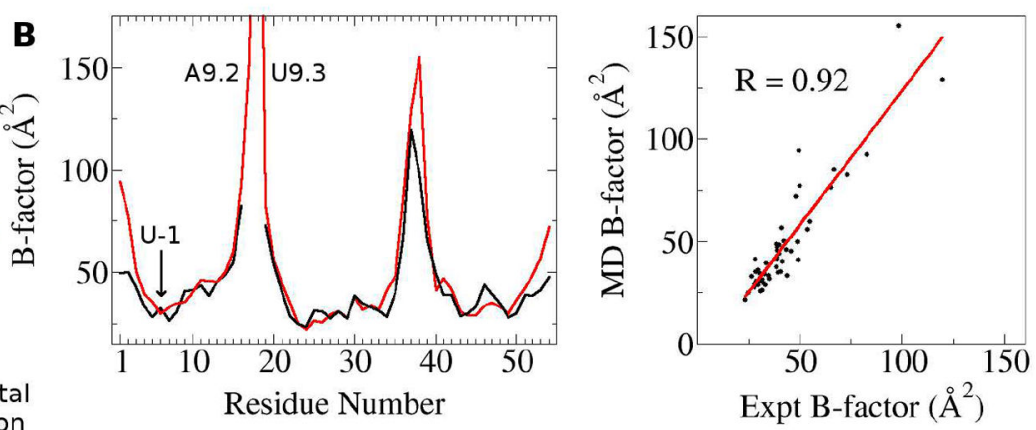

D

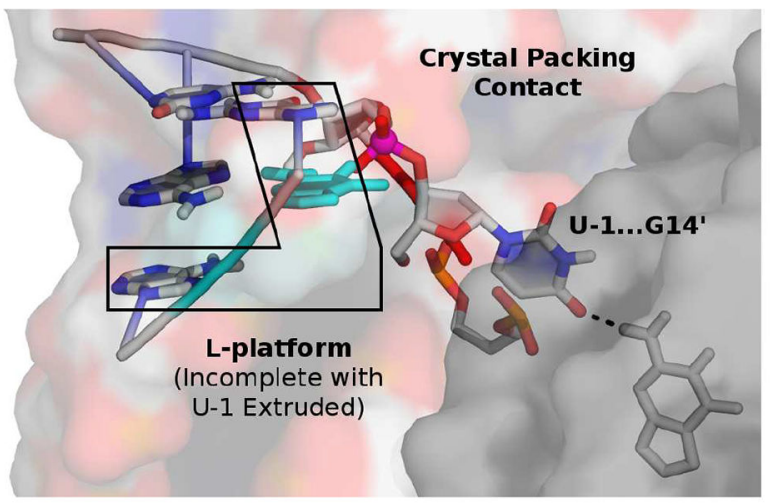

Figure 3: Crystal Simulation of PDB ID: 4OJI twister ribozyme.

(a) Unit cell of 4OJI structure containing 12 asymmetric units used for crystal simulation viewed along six-fold symmetry axis. (b) Comparison of simulated and experimental Bfactors, from $270 \mathrm{~ns}$ of MD crystal simulation. B-factors are calculated for each residue from a single combined trajectory where the full ensemble of structures (12 asymmetric units) is considered after applying the appropriate symmetry operations. (c) Overlay of average simulation (colored) and experimental (black) structures for the active site residues considered as part of the L-platform/L-anchor motif. (d) Crystal packing contact between U-1 and G14' that stabilizes the conformation of U-1 where it is displaced from the heel of the L-platform. 

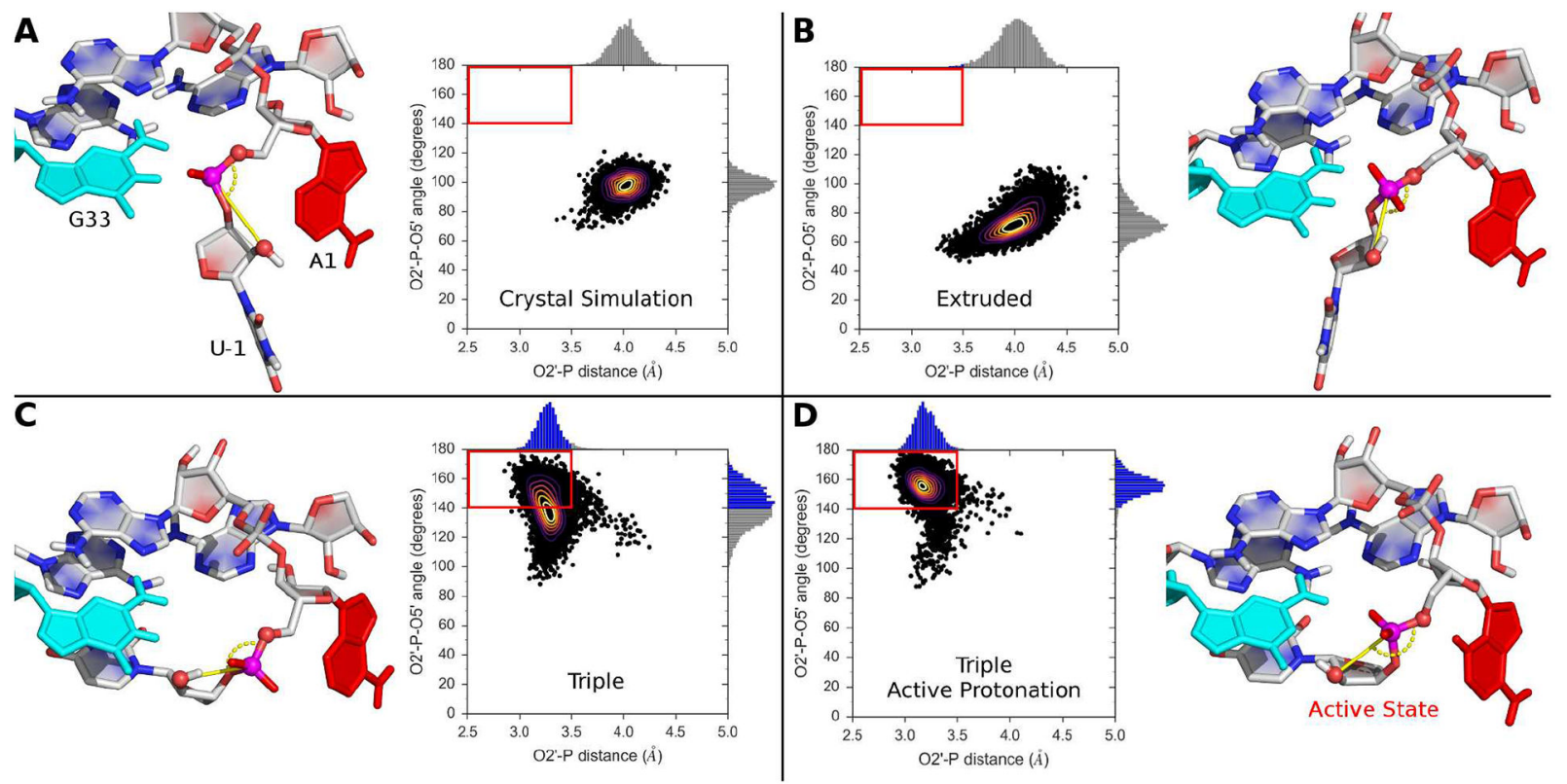

Figure 4: In-line fitness of the twister ribozyme.

Plots of nucleophilic attack parameters: $02^{\prime}-\mathrm{P}-05^{\prime}$ angle vs. $02^{\prime}$-P distance, with the red box in the upper left corner indicating high "in-line fitness". Representative structures corresponding to each plot are shown, high-lighting the alignment of the active site residues and scissile phosphate. Reactive atoms shown as spheres (oxygens in red and phosphate in magenta). General acid and base colored light blue and red, respectively. (a) Results from $270 \mathrm{~ns}$ crystal simulation of the 4OJI structure, the nucleophile was modeled in at each frame for analysis alone. (b-d) Results for the 4OJI sequence in a solution environment with the nucleophile included in the $75 \mathrm{~ns}$ simulation, where U-1 is restrained to either be (b) extruded from the active site (similar to the crystal), or (c,d) forming a base triple within the L1 loop. In simulations a-c the presumptive acid and base are in the neutral protonation state, whereas the results shown in panel $\mathbf{d}$ are from a simulation of the active state where the $\mathrm{G} 33$ is deprotonated at the $\mathrm{N} 1$ position and $\mathrm{A} 1$ is protonated at the $\mathrm{N} 3$ position. Contours are drawn to highlight the density of overlapping data points, while the corresponding distributions are colored blue for angles or distances within the region indicating high "inline fitness" $\left(02^{\prime}-\mathrm{P}-05^{\prime}\right.$ angle $>140^{\circ}$ and $02^{\prime}$-P distance $<3.5 \AA$ ). 
A

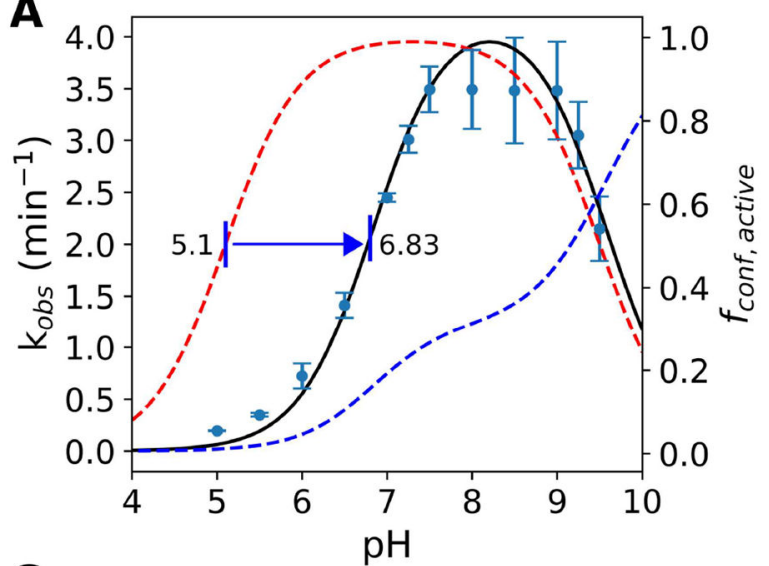

$\mathbf{E}$

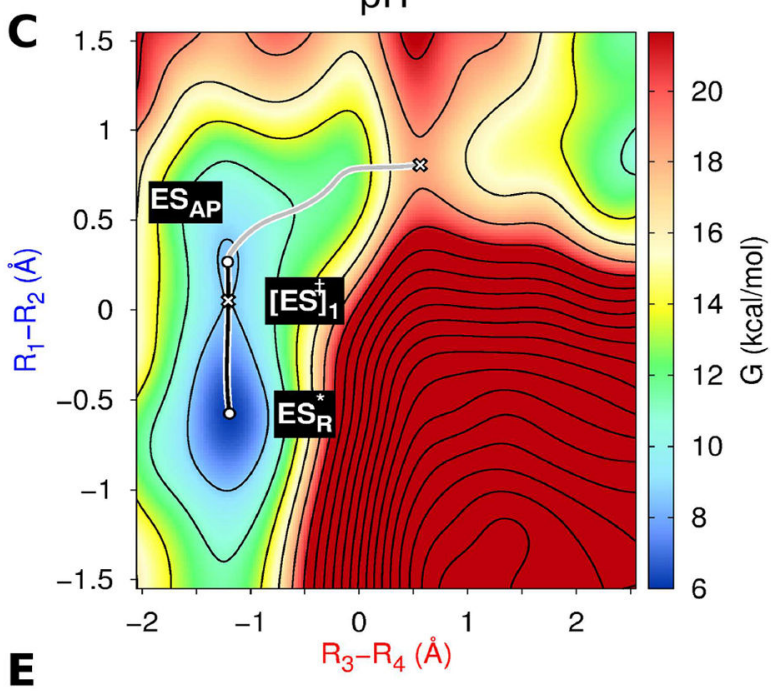

Activity-pH:

$\Delta G$ in

$\mathrm{kcal} / \mathrm{mol}$
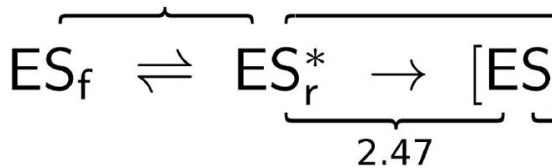

$\mathrm{QM} / \mathrm{MM}$ :
B

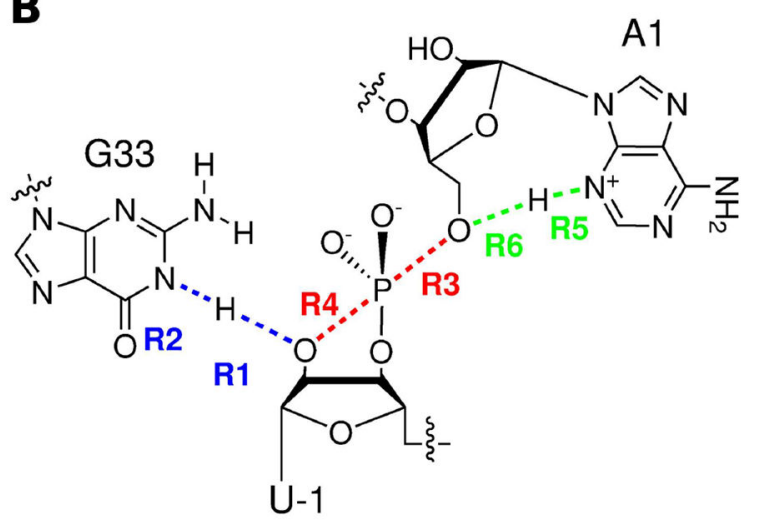

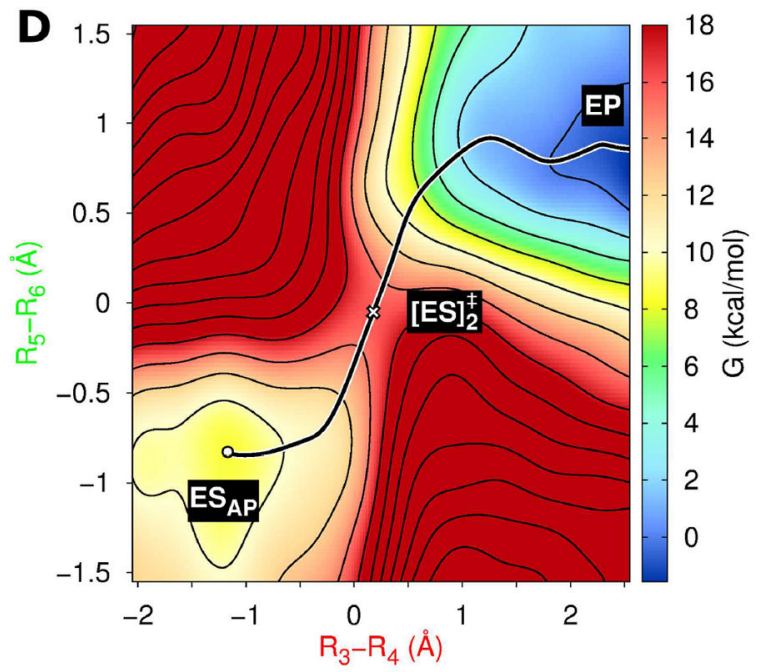

$\leq 12.61$

Figure 5: Model for catalytic mechanism of the twister ribozyme.

(a) Experimental activity-pH profile. ${ }^{31}$ Black: Conformational influencer model fit to the experimental data for the WT twister construct. ${ }^{31}$ Red: Cooperative model (scaled) with $p K_{a}$ values assumed to be 5.1 and 9.5 with 0.21 units of coupling. Blue: Fraction of the active conformational state for the $\mathrm{pH}$-dependent L-platform formation that produces an apparent $p K_{a}$ shift of the general acid from 5.1 to 6.83. (b) QM/MM reaction coordinates for: General base (R1-R2, blue), Phosphoryl transfer (R3-R4, red) and General acid (R5-R6, green) steps. (c, d) 2D ab initio QM/MM free energy profile for (c) the general base and phosphoryl transfer steps, and (d) general acid and phosphoryl transfer steps. The two profiles intersect at the local free energy minimum $\left(E S_{A P}\right)$, and together indicate a stepwise nucleophile activation followed by a concerted nucleophilic substitution with partial proton transfer in the rate-controlling transition state. (e) Estimated free energies $(\mathrm{kcal} / \mathrm{mol})$ for the proposed 
catalytic mechanism from both the conformational influencer model (a) and the QM/MM simulations $(\mathbf{c}, \mathbf{d})$. 


\begin{tabular}{|c|c|c|c|c|c|c|}
\hline & $\begin{array}{l}\text { WT } \\
(9.5)\end{array}$ & (10.6) & ${ }_{\mathrm{H}}^{\mathrm{G} 33(6 \mathrm{sG})}{ }_{\mathrm{NH}}^{(8.7)}$ & (3.6) & (3) & ${ }_{\mathrm{H}+05 \cdot \mathrm{S}}^{\mathrm{Al}(3 \mathrm{CA})}$ \\
\hline $\begin{array}{l}\Delta \mathrm{G}_{\text {active }} \\
\Delta \mathrm{G}_{\text {int }} \\
\Delta \mathrm{G}_{\text {obs }}\end{array}$ & $\begin{array}{r}\geq \quad 6.18 \\
9.63 \\
\geq 15.81\end{array}$ & $\begin{array}{r}\geq \quad 7.68 \\
9.39 \\
\geq 17.07\end{array}$ & $\begin{array}{r}\geq \quad 5.09 \\
12.04 \\
\geq 17.13\end{array}$ & $\begin{array}{r}\geq 2.31 \\
15.94 \\
\geq 18.25\end{array}$ & $\begin{array}{r}\geq \quad 3.87 \\
29.99 \\
\geq 33.86\end{array}$ & $\begin{array}{r}\geq \quad 3.87 \\
5.19 \\
\geq 9.06\end{array}$ \\
\hline $\begin{array}{l}\mathrm{k}_{o b s} \\
\mathrm{k} / \mathrm{k}^{\prime}\end{array}$ & $\begin{array}{r}\leq 800 \\
\ldots\end{array}$ & $\begin{array}{r}\leq 93 \\
8.5\end{array}$ & $\begin{array}{r}85 \\
9\end{array}$ & $\begin{array}{r}\leq 13 \\
63\end{array}$ & $\begin{array}{r}\mathrm{nd}\left(\leq 10^{-7}\right) \\
\ldots\end{array}$ & $\begin{array}{r}\leq 7.6 \times 10^{7} \\
1.0 \times 10^{-4}\end{array}$ \\
\hline
\end{tabular}

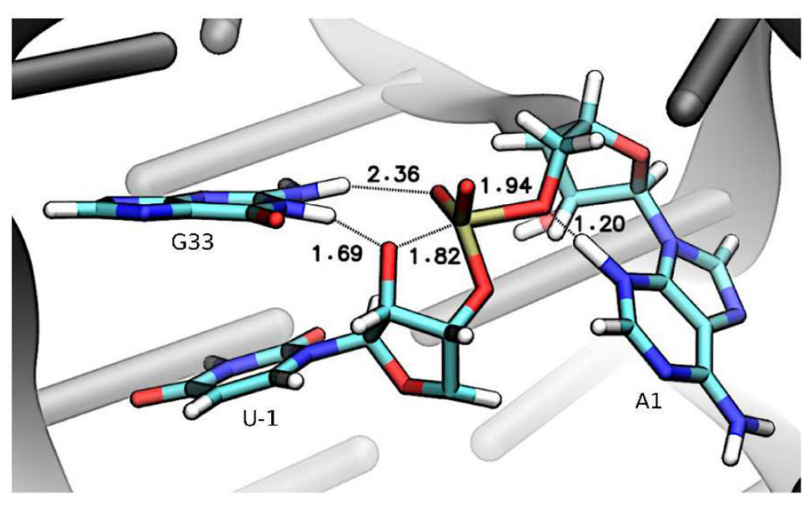

${ }^{18} \mathrm{O}$ Kinetic Isotope Effects

\begin{tabular}{lrrr}
\hline Non-Enzymatic & ${ }^{18} k_{N U C}$ & ${ }^{18} k_{L G}$ & ${ }^{18} k_{N P O}$ \\
\hline Calc. & 0.977 & 1.042 & 1.004 \\
Expt. & 0.984 & 1.034 & 0.999
\end{tabular}

RNase A

\begin{tabular}{llll}
\hline Calc. & 0.993 & 1.028 & 1.002
\end{tabular}

Expt. $\quad 0.994 \quad 1.014 \quad 1.001$

Twister

Calc.

$0.997 \quad 1.002$

Figure 6: Experimental predictions of mutational and kinetic isotope effects.

(Top) Predictions of mutational effects on the balance of free energy for adoption of the catalytically active state in solution and the intrinsic rate of reaction. $\Delta \mathrm{G}_{\text {active }}$ is derived at pH 7 from the conformational influencer model and thus only provides a lower bound, while $\Delta \mathrm{G}_{\text {int }}$ is derived from the QM/MM simulations. For the WT and general base mutants the predicted $\mathrm{pK}_{a}$ for the $\mathrm{N} 1$ of residue 33 is listed in parentheses. Free energies are in $\mathrm{kcal} / \mathrm{mol}$, while rate constants have units of $\min ^{-1}$ (Bottom Left) The PBE0/6-31G* optimized transition state structure of twister ribozyme. (Bottom Right) Predictions of kinetic isotope effect values calculated at the PBE0/6-31G* level of theory along with experimental values for the non-enzymatic model reaction and RNase A. 59 